\title{
The Algebraic Cluster Model: Structure of ${ }^{16} \mathrm{O}$
}

\author{
R. Bijker \\ Instituto de Ciencias Nucleares, Universidad Nacional Autónoma de México, \\ Apartado Postal 70-543, 04510 México, D.F., México \\ F. Iachello \\ Center for Theoretical Physics, Sloane Laboratory, Yale University, \\ New Haven, CT 06520-8120, U.S.A.
}

\begin{abstract}
We discuss an algebraic treatment of four-body clusters which includes both continuous and discrete symmetries. In particular, tetrahedral configurations with $\mathcal{T}_{d}$ symmetry are analyzed with respect to the energy spectrum, transition form factors and $B(E L)$ values. It is concluded that the low-lying spectrum of ${ }^{16} \mathrm{O}$ can be described by four $\alpha$ particles at the vertices of a regular tetrahedron, not as a rigid structure but rather a more floppy structure with relatively large rotation-vibration interactions and Coriolis forces.
\end{abstract}

Keywords: Cluster model, Alpha-cluster nuclei, Algebraic models

\section{Introduction}

The binding energy per nucleon for light nuclei shows large oscillations with nucleon number with maxima for nuclei with $A=4 n$ and $Z=N$, especially for the nuclei ${ }^{4} \mathrm{He},{ }^{8} \mathrm{Be},{ }^{12} \mathrm{C}$ and ${ }^{16} \mathrm{O}$ for $n=1,2,3$ and 4 , respectively, which provides a strong indication of the importance of $\alpha$ clustering in these nuclei. The phenomenon of clustering in light nuclei has a large history dating back to the 1930's with early studies on $\alpha$-cluster models by Wheeler [1], and Hafstad and Teller [2], followed by later work by Dennison [3], Kameny [4], Brink [5, 6] and Robson [7,8]. The connection between the nuclear shell model and the cluster model was studied in [9], as well as by the the Japanese school [10, 11, 12, 13]. A recent review on cluster models can be found in Ref. [14].

In the last few years, there has been considerable renewed interest in the structure of $\alpha$-cluster nuclei, especially for the nucleus ${ }^{12} \mathrm{C}$ [15]. The measurement of new rotational excitations of both the ground state $[16,17,18]$ and the

Email addresses: bijker@nucleares.unam.mx (R. Bijker), francesco.iachello@yale.edu (F. Iachello) 
Hoyle state $[19,20,21,22]$ has stimulated a large effort to understand the structure of ${ }^{12} \mathrm{C}$ ranging from studies based on antisymmetrized molecular dynamics (AMD) [23], fermionic molecular dynamics (FMD) [24], BEC-like cluster model [25], ab initio no-core shell model [26], lattice EFT [27, 28], no-core symplectic model [29] and the algebraic cluster model (ACM) [18, 30, 31, 32].

The aim of this paper is to develop the ACM for four-body cluster systems and especially to discuss an application to the nucleus ${ }^{16} \mathrm{O}$ as a cluster of four $\alpha$-particles. The ACM provides an algebraic treatment of the relative motion of the clusters in which eigenvalues and eigenfunctions are obtained by matrix diagonalization instead of by solving a set of coupled differential equations. Algebraic models have found useful applications both in many- and in fewbody systems. As an example we mention the interacting boson model (IBM), which has been very successful in the description of collective states in nuclei [33]. Its dynamical symmetries correspond to the quadrupole vibrator [34], the axially symmetric rotor [35] and the $\gamma$-unstable rotor [36] in a geometrical description. The first extension to few-body systems was the vibron model [37] which was introduced to describe vibrational and rotational excitations in diatomic molecules [38]. The dynamical symmetries of the vibron model correspond to the (an)harmonic oscillator and the Morse oscillator.

The general procedure is to introduce a $U(k+1)$ spectrum generating algebra for a bound-state problem with $k$ degrees of freedom [39]. For the five quadrupole degrees of freedom in collective nuclei this led to the introduction of the $U(6)$ interacting boson model [33]. Similarly, the $U(4)$ vibron model was proposed to describe the dynamics of the three dipole degrees of freedom of the relative motion of two objects, e.g. two atoms in a diatomic molecule [37], two clusters in a nuclear cluster model $[40,41,42]$, or quark-antiquark configurations in mesons $[43,44]$. For three-body cluster systems the algebra is $U(7)$. The $U(7)$ algebraic cluster model was developed originally to describe the relative motion of the three constituent quarks in baryons $(q q q)[45,46]$, but has also found applications in molecular physics $\left(\mathrm{H}_{3}^{+}\right)[47,48]$ and nuclear physics $\left({ }^{12} \mathrm{C}\right.$ as a cluster of three $\alpha$ particles) $[30,31]$.

This paper is organized as follows. In Sects. 2-3, we introduce the algebraic cluster model for four-body systems in terms of a $U(10)$ spectrum-generating algebra, followed by a discussion of the permutation symmetry for the case of four identical objects. In Sect. 4, we show that within $U(10)$ it is possible to provide a description of a spherical top with tetrahedral symmetry in which all vibrational and rotational degrees of freedom are present from the outset. In Sect. 5, we discuss how to calculate transition probabilities in the ACM and we apply the $U(10)$ model in the limit of the spherical top to the description of the nucleus ${ }^{16} \mathrm{O}$ as a cluster of four $\alpha$-particles, and show evidence for $\mathcal{T}_{d}$ symmetry in this nucleus.

A preliminary account of part of this work has appeared in Refs. [32, 49, 50, 51]. Here we develop the $U(10) \mathrm{ACM}$ in more detail, and especially we present a detailed analysis of the spectroscopic properties of ${ }^{16} \mathrm{O}$ including energies, transition rates and electromagnetic form factors, and show that ${ }^{16} \mathrm{O}$ can be, to a good approximation, described by a $4 \alpha$ configuration with $\mathcal{T}_{d}$ symmetry. 
The development of the $U(10) \mathrm{ACM}$ described here opens the way for applications to systems with generic geometric configurations as mentioned in the final Sect. 7.

\section{Algebraic Cluster Model}

The Algebraic Cluster Model (ACM) is a model designed to describe the relative motion of a cluster system. We start by introducing the relative Jacobi coordinates for a four-body system, see Fig. 1,

$$
\begin{aligned}
& \vec{\rho}=\left(\vec{r}_{1}-\vec{r}_{2}\right) / \sqrt{2}, \\
& \vec{\lambda}=\left(\vec{r}_{1}+\vec{r}_{2}-2 \vec{r}_{3}\right) / \sqrt{6}, \\
& \vec{\eta}=\left(\vec{r}_{1}+\vec{r}_{2}+\vec{r}_{3}-3 \vec{r}_{4}\right) / \sqrt{12},
\end{aligned}
$$

together with their conjugate momenta. Here $\vec{r}_{i}(i=1, \ldots, 4)$ represent the coordinates of the four constituent particles. Instead of a formulation in terms of coordinates and momenta we use the method of bosonic quantization which consists in quantizing the Jacobi coordinates and momenta with vector boson operators and adding an additional scalar boson

$$
b_{\rho m}^{\dagger}, b_{\lambda m}^{\dagger}, b_{\eta m}^{\dagger}, s^{\dagger}, \quad(m=-1,0,1),
$$

altoghether denoted as $c_{i}^{\dagger}$ with $i=1 \ldots, 10$, under the constraint that the Hamiltonian commutes with the number operator

$$
\hat{N}=\sum_{i} c_{i}^{\dagger} c_{i}=s^{\dagger} s+\sum_{m}\left(b_{\rho m}^{\dagger} b_{\rho m}+b_{\lambda m}^{\dagger} b_{\lambda m}+b_{\eta m}^{\dagger} b_{\eta m}\right),
$$

i.e. the total number of bosons $N=n_{s}+n_{\rho}+n_{\lambda}+n_{\eta}$ is conserved. The set of 100 bilinear products of creation and annihilation operators $G_{i j}=c_{i}^{\dagger} c_{j}$ spans the Lie algebra of $U(10)$. All operators of interest, such as the Hamiltonian and electromagnetic transition operators, are expressed in terms of elements of this algebra. As an example, the one- and two-body Hamiltonian is given by

$$
H=\sum_{i j} \epsilon_{i j} G_{i j}+\sum_{i j k l} v_{i j k l} G_{i j} G_{k l} .
$$

The model space of the ACM is spanned by the symmetric irreducible representation $[N]$ of $U(10)$ which contains the oscillator shells with $n=n_{\rho}+n_{\lambda}+n_{\eta}=$ $0,1, \ldots, N$. The introduction of the scalar boson makes it possible to investigate the dynamics of three vector degrees of freedom including situations in which there is a mixing of oscillator shells. It can be seen as a convenient way to compactify the infinite dimensional space of the harmonic oscillator to a finite-dimensional space. 


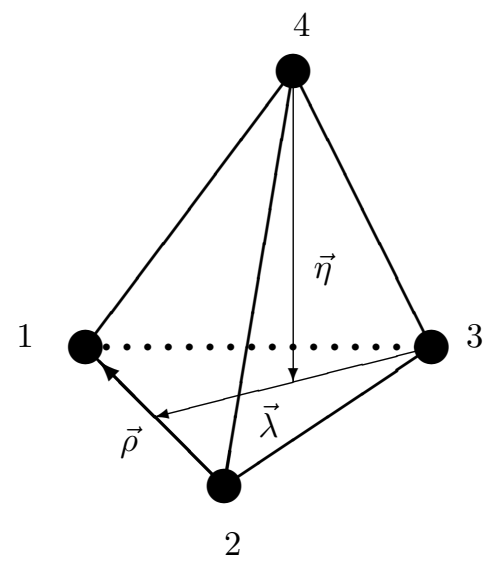

Figure 1: Jacobi coordinates in a tetrahedral configuration

\section{Permutation symmetry}

In case of identical clusters, the Hamiltonian should be invariant under their permutation and, as a consequence, the states would transform according to the representations of the corresponding permutation group.

For four identical objects, as for example for $\mathrm{X}_{4}$ molecules or $4 \alpha$ clusters, the Hamiltonian has to be invariant under the permuation group $S_{4}$. The permutation symmetry of four identical objects is determined by the transposition $P(12)$ and the cyclic permutation $P(1234)$ [52]. All other permutations can be expressed in terms of these two elementary ones. The transformation properties under $S_{4}$ of all operators in the model follow from those of the building blocks. Algebraically, the transposition and cyclic permutation can be expressed in terms of the generators $b_{i}^{\dagger} b_{j} \equiv \sum_{m} b_{i, m}^{\dagger} b_{j, m}$ that act in index space $(i, j=\rho$, $\lambda, \eta)$. The transposition is given by

$$
P(12)\left(\begin{array}{c}
s^{\dagger} \\
b_{\rho}^{\dagger} \\
b_{\lambda}^{\dagger} \\
b_{\eta}^{\dagger}
\end{array}\right)=U_{\mathrm{tr}}\left(\begin{array}{c}
s^{\dagger} \\
b_{\rho}^{\dagger} \\
b_{\lambda}^{\dagger} \\
b_{\eta}^{\dagger}
\end{array}\right) U_{\mathrm{tr}}^{-1}=\left(\begin{array}{rrrr}
1 & 0 & 0 & 0 \\
0 & -1 & 0 & 0 \\
0 & 0 & 1 & 0 \\
0 & 0 & 0 & 1
\end{array}\right)\left(\begin{array}{c}
s^{\dagger} \\
b_{\rho}^{\dagger} \\
b_{\lambda}^{\dagger} \\
b_{\eta}^{\dagger}
\end{array}\right),
$$

with

$$
U_{\mathrm{tr}}=\mathrm{e}^{i \pi b_{\rho}^{\dagger} b_{\rho}},
$$


and the cyclic permutation by

$$
\begin{aligned}
P(1234)\left(\begin{array}{c}
s^{\dagger} \\
b_{\rho}^{\dagger} \\
b_{\lambda}^{\dagger} \\
b_{\eta}^{\dagger}
\end{array}\right) & =U_{\mathrm{cycl}}\left(\begin{array}{c}
s^{\dagger} \\
b_{\rho}^{\dagger} \\
b_{\lambda}^{\dagger} \\
b_{\eta}^{\dagger}
\end{array}\right) U_{\mathrm{cycl}}^{-1} \\
& =\left(\begin{array}{cccc}
1 & 0 & 0 & 0 \\
0 & -\frac{1}{2} & \frac{\sqrt{3}}{2} & 0 \\
0 & -\frac{1}{2 \sqrt{3}} & -\frac{1}{6} & \frac{\sqrt{8}}{3} \\
0 & -\frac{\sqrt{2}}{\sqrt{3}} & -\frac{\sqrt{2}}{3} & -\frac{1}{3}
\end{array}\right)\left(\begin{array}{c}
s^{\dagger} \\
b_{\rho}^{\dagger} \\
b_{\lambda}^{\dagger} \\
b_{\eta}^{\dagger}
\end{array}\right),
\end{aligned}
$$

with

$$
U_{\text {cycl }}=\mathrm{e}^{i \pi\left(b_{\rho}^{\dagger} b_{\rho}+b_{\lambda}^{\dagger} b_{\lambda}+b_{\eta}^{\dagger} b_{\eta}\right)} \mathrm{e}^{\theta_{1}\left(b_{\rho}^{\dagger} b_{\lambda}-b_{\lambda}^{\dagger} b_{\rho}\right)} \mathrm{e}^{\theta_{2}\left(b_{\lambda}^{\dagger} b_{\eta}-b_{\eta}^{\dagger} b_{\lambda}\right)},
$$

and $\theta_{1}=\arctan \sqrt{3}$ and $\theta_{2}=\arctan \sqrt{8}$. The scalar boson, $s^{\dagger}$, transforms as the symmetric representation [4], whereas the three vector Jacobi bosons, $b_{\rho}^{\dagger}, b_{\lambda}^{\dagger}$ and $b_{\eta}^{\dagger}$, transform as the three components of the mixed symmetry representation [31].

There are five different symmetry classes for the permutation of four objects. Since $S_{4}$ is isomorphic to the tetrahedral group $\mathcal{T}_{d}$, the irreducible representations can also be labeled by those of the tetrahedral group

$$
\begin{aligned}
{[4] } & \sim A_{1}, \\
{[31] } & \sim F_{2}, \\
{[22] } & \sim E \\
{[211] } & \sim F_{1}, \\
{[1111] } & \sim A_{2} .
\end{aligned}
$$

In the remainder of this article we will use the irreducible representations of $\mathcal{T}_{d}$ to indicate the tensorial character under the permutation group.

Next, one can use the multiplication rules for $\mathcal{I}_{d}$ to construct physical operators with the appropriate symmetry properties. For example, for the bilinear products of the three vector Jacobi bosons, one finds

$$
F_{2} \otimes F_{2}=A_{1} \oplus F_{2} \oplus F_{1} \oplus E .
$$

It is convenient to express the 100 elements of the algebra of $U(10)$ in terms of tensor operators under both the rotation group $S O(3)$ and the tetrahedral 
group $\mathcal{T}_{d} \sim S_{4}$ as

$$
\begin{aligned}
& A_{1} \quad \hat{n}_{s}=\left(s^{\dagger} \tilde{s}\right)_{0}^{(0)} \\
& F_{2} \quad\left\{\begin{array}{l}
\hat{D}_{\rho}=\left(b_{\rho}^{\dagger} \tilde{s}-s^{\dagger} \tilde{b}_{\rho}\right)^{(1)} \\
\hat{D}_{\lambda}=\left(b_{\lambda}^{\dagger} \tilde{s}-s^{\dagger} \tilde{b}_{\lambda}\right)^{(1)} \\
\hat{D}_{\eta}=\left(b_{\eta}^{\dagger} \tilde{s}-s^{\dagger} \tilde{b}_{\eta}\right)^{(1)}
\end{array}\right. \\
& F_{2} \quad\left\{\begin{array}{l}
\hat{A}_{\rho}=i\left(b_{\rho}^{\dagger} \tilde{s}+s^{\dagger} \tilde{b}_{\rho}\right)^{(1)} \\
\hat{A}_{\lambda}=i\left(b_{\lambda}^{\dagger} \tilde{s}+s^{\dagger} \tilde{b}_{\lambda}\right)^{(1)} \\
\hat{A}_{\eta}=i\left(b_{\eta}^{\dagger} \tilde{s}+s^{\dagger} \tilde{b}_{\eta}\right)^{(1)}
\end{array}\right. \\
& A_{1} \quad \hat{B}^{(l)}=\left(b_{\rho}^{\dagger} \tilde{b}_{\rho}+b_{\lambda}^{\dagger} \tilde{b}_{\lambda}+b_{\eta}^{\dagger} \tilde{b}_{\eta}\right)^{(l)} \\
& E \quad\left\{\begin{array}{l}
\hat{C}_{\rho}^{(l)}=\left(b_{\rho}^{\dagger} \tilde{b}_{\lambda}+b_{\lambda}^{\dagger} \tilde{b}_{\rho}\right)^{(l)}-\sqrt{2}\left(b_{\rho}^{\dagger} \tilde{b}_{\eta}+b_{\eta}^{\dagger} \tilde{b}_{\rho}\right)^{(l)} \\
\hat{C}_{\lambda}^{(l)}=\left(b_{\rho}^{\dagger} \tilde{b}_{\rho}-b_{\lambda}^{\dagger} \tilde{b}_{\lambda}\right)^{(l)}-\sqrt{2}\left(b_{\lambda}^{\dagger} \tilde{b}_{\eta}+b_{\eta}^{\dagger} \tilde{b}_{\lambda}\right)^{(l)}
\end{array}\right. \\
& F_{2} \quad\left\{\begin{array}{l}
\hat{G}_{\rho}^{(l)}=\sqrt{2}\left(b_{\rho}^{\dagger} \tilde{b}_{\lambda}+b_{\lambda}^{\dagger} \tilde{b}_{\partial}\right)^{(l)}+\left(b_{\rho}^{\dagger} \tilde{b}_{\eta}+b_{\eta}^{\dagger} \tilde{b}_{\partial}\right)^{(l)} \\
\hat{G}_{\lambda}^{(l)}=\sqrt{2}\left(b_{\rho}^{\dagger} \tilde{b}_{\rho}-b_{\lambda}^{\dagger} \tilde{b}_{\lambda}\right)^{(l)}+\left(b_{\lambda}^{\dagger} \tilde{b}_{\eta}+b_{\eta}^{\dagger} \tilde{b}_{\lambda}\right)^{(l)} \\
\hat{G}_{\eta}^{(l)}=\left(b_{\rho}^{\dagger} \tilde{b}_{\rho}+b_{\lambda}^{\dagger} \tilde{b}_{\lambda}-2 b_{\eta}^{\dagger} \tilde{b}_{\eta}\right)^{(l)}
\end{array}\right. \\
& F_{1} \quad\left\{\begin{array}{l}
\hat{K}_{\rho}^{(l)}=-i\left(b_{\lambda}^{\dagger} \tilde{b}_{\eta}-b_{\eta}^{\dagger} \tilde{b}_{\lambda}\right)^{(l)} \\
\hat{K}_{\lambda}^{(l)}=-i\left(b_{\eta}^{\dagger} \tilde{b}_{\rho}-b_{\rho}^{\dagger} \tilde{b}_{\eta}\right)^{(l)} \\
\hat{K}_{\eta}^{(l)}=-i\left(b_{\rho}^{\dagger} \tilde{b}_{\lambda}-b_{\lambda}^{\dagger} \tilde{b}_{\rho}\right)^{(l)}
\end{array}\right.
\end{aligned}
$$

with $l=0,1,2$. Here $\tilde{s}=s$ and $\tilde{b}_{k m}=(-1)^{1-m} b_{k,-m}$ where $k$ denotes the three Jacobi coordinates $\rho, \lambda, \eta$.

The invariance under tetrahedral symmetry imposes strong constraints on the general Hamiltonian. The most general one- and two-body Hamiltonian that is rotationally invariant, conserves parity as well as the total number of bosons, and in addition is scalar under the tetrahedral group $\mathcal{I}_{d} \sim S_{4}$, is given by

$$
\begin{aligned}
H= & \epsilon_{0} s^{\dagger} \tilde{s}-\epsilon_{1}\left(b_{\rho}^{\dagger} \cdot \tilde{b}_{\rho}+b_{\lambda}^{\dagger} \cdot \tilde{b}_{\lambda}+b_{\eta}^{\dagger} \cdot \tilde{b}_{\eta}\right) \\
& +u_{0} s^{\dagger} s^{\dagger} \tilde{s} \tilde{s}-u_{1} s^{\dagger}\left(b_{\rho}^{\dagger} \cdot \tilde{b}_{\rho}+b_{\lambda}^{\dagger} \cdot \tilde{b}_{\lambda}+b_{\eta}^{\dagger} \cdot \tilde{b}_{\eta}\right) \tilde{s} \\
+ & v_{0}\left[\left(b_{\rho}^{\dagger} \cdot b_{\rho}^{\dagger}+b_{\lambda}^{\dagger} \cdot b_{\lambda}^{\dagger}+b_{\eta}^{\dagger} \cdot b_{\eta}^{\dagger}\right) \tilde{s} \tilde{s}+\text { h.c. }\right] \\
+\sum_{L=0,2} a_{L}[ & {\left[2 b_{\rho}^{\dagger} b_{\eta}^{\dagger}+2 \sqrt{2} b_{\rho}^{\dagger} b_{\lambda}^{\dagger}\right]^{(L)} \cdot[\text { h.c. }]^{(L)} } \\
& +\left[2 b_{\lambda}^{\dagger} b_{\eta}^{\dagger}+\sqrt{2}\left(b_{\rho}^{\dagger} b_{\rho}^{\dagger}-b_{\lambda}^{\dagger} b_{\lambda}^{\dagger}\right)\right]^{(L)} \cdot[\text { h.c. }]^{(L)} \\
& \left.+\left[b_{\rho}^{\dagger} b_{\rho}^{\dagger}+b_{\lambda}^{\dagger} b_{\lambda}^{\dagger}-2 b_{\eta}^{\dagger} b_{\eta}^{\dagger}\right]^{(L)} \cdot[\text { h.c. }]^{(L)}\right]
\end{aligned}
$$




$$
\begin{gathered}
+\sum_{L=0,2} c_{L}\left[\left[-2 \sqrt{2} b_{\rho}^{\dagger} b_{\eta}^{\dagger}+2 b_{\rho}^{\dagger} b_{\lambda}^{\dagger}\right]^{(L)} \cdot[\text { h.c. }]^{(L)}\right. \\
\left.+\left[-2 \sqrt{2} b_{\lambda}^{\dagger} b_{\eta}^{\dagger}+\left(b_{\rho}^{\dagger} b_{\rho}^{\dagger}-b_{\lambda}^{\dagger} b_{\lambda}^{\dagger}\right)\right]^{(L)} \cdot[\text { h.c. }]^{(L)}\right] \\
+c_{1}\left[\left(b_{\rho}^{\dagger} b_{\lambda}^{\dagger}\right)^{(1)} \cdot\left(\tilde{b}_{\lambda} \tilde{b}_{\rho}\right)^{(1)}+\left(b_{\lambda}^{\dagger} b_{\eta}^{\dagger}\right)^{(1)} \cdot\left(\tilde{b}_{\eta} \tilde{b}_{\lambda}\right)^{(1)}+\left(b_{\eta}^{\dagger} b_{\rho}^{\dagger}\right)^{(1)} \cdot\left(\tilde{b}_{\rho} \tilde{b}_{\eta}\right)^{(1)}\right] \\
+\sum_{L=0,2} d_{L}\left(b_{\rho}^{\dagger} b_{\rho}^{\dagger}+b_{\lambda}^{\dagger} b_{\lambda}^{\dagger}+b_{\eta}^{\dagger} b_{\eta}^{\dagger}\right)^{(L)} \cdot(\text { h.c. })^{(L)} .
\end{gathered}
$$

By construction, the wave functions are characterized by the total number of bosons $N$, angular momentum $L$ and parity $P$, and their transformation property $t$ under the tetrahedral group. Since we do not consider internal excitations of the clusters, the four-body wave functions arise solely from the relative motion and are symmetric with $t=A_{1}$.

The relation between harmonic oscillators and permutation symmetry was studied by Kramer and Moshinsky [52]. In the present case, we wish to study the properties of the $U(10)$ algebraic cluster model for any (large) number of oscillator quanta with the possibility of mixing between different oscillator shells. Therefore, we prefer to generate a set of basis states with good permutation symmetry numerically by diagonalization of $\mathcal{T}_{d} \sim S_{4}$ invariant interactions. The permutation symmetry $t$ of a given eigenfunction can then be determined from the transformation properties under the transposition $P(12)$ and the cyclic permutation $P(1234)$ [52]. In practice, the wave functions are obtained numerically by diagonalization, and hence are determined up to a sign. The relative phases of the degenerate representations, the two-dimensional $E$, and the threedimensional $F_{2}$ and $F_{1}$, can be determined from the off-diagonal matrix elements of $P(1234)$ [51].

\section{Special solutions}

In general, the eigenvalues and corresponding eigenvectors are obtained numerically by diagonalizing the Hamiltonian of Eq. (12) in a coupled harmonic oscillator basis. However, there are special limiting cases of the Hamiltonian of Eq. (12), in which the energy spectra can be obtained in closed form. These special cases are called dynamical symmetries and arise whenever the Hamiltonian is expressed in terms of the Casimir invariants of a chain of subalgebras of $U(10)$. Two examples of dynamical symmetries of the $S_{4} \sim \mathcal{T}_{d}$ invariant Hamiltonian correspond to the group lattice

$$
U(10) \supset\left\{\begin{array}{c}
U(9) \\
S O(10)
\end{array}\right\} \supset S O(9) \supset \mathcal{S O}(3) \otimes S O(3),
$$

where $\mathcal{S O}(3)$ denotes the angular momentum group in coordinate space and $S O(3)$ the angular momentum in index space. These dynamical symmetries were shown to correspond to the nine-dimensional (an)harmonic oscillator and the nine-dimensional deformed oscillator, respectively $[49,50,51]$. 
In the following, we discuss in more detail the case of the spherical top. Although it does not correspond to a dynamical symmetry, approximate solutions can still be obtained in the large $N$ limit, which subsequently will be used to analyze and interpret the cluster states in ${ }^{16} \mathrm{O}$. An interesting limiting case of the general $\mathcal{T}_{d}$ invariant Hamiltonian of Eq. (12) is provided by [32]

$$
\begin{aligned}
H_{3, \mathrm{vib}}= & \xi_{1}\left(R^{2} s^{\dagger} s^{\dagger}-b_{\rho}^{\dagger} \cdot b_{\rho}^{\dagger}-b_{\lambda}^{\dagger} \cdot b_{\lambda}^{\dagger}-b_{\eta}^{\dagger} \cdot b_{\eta}^{\dagger}\right) \text { (h.c.) } \\
+ & \xi_{2}\left[\left(-2 \sqrt{2} b_{\rho}^{\dagger} \cdot b_{\eta}^{\dagger}+2 b_{\rho}^{\dagger} \cdot b_{\lambda}^{\dagger}\right)(\text { h.c. })\right. \\
& \left.\left.+\left(-2 \sqrt{2} b_{\lambda}^{\dagger} \cdot b_{\eta}^{\dagger}+\left(b_{\rho}^{\dagger} \cdot b_{\rho}^{\dagger}-b_{\lambda}^{\dagger} \cdot b_{\lambda}^{\dagger}\right)\right) \text { (h.c. }\right)\right] \\
+\xi_{3}\left[\left(2 b_{\rho}^{\dagger} \cdot b_{\eta}^{\dagger}\right.\right. & \left.+2 \sqrt{2} b_{\rho}^{\dagger} \cdot b_{\lambda}^{\dagger}\right)(\text { h.c. }) \\
& +\left(2 b_{\lambda}^{\dagger} \cdot b_{\eta}^{\dagger}+\sqrt{2}\left(b_{\rho}^{\dagger} \cdot b_{\rho}^{\dagger}-b_{\lambda}^{\dagger} \cdot b_{\lambda}^{\dagger}\right)\right)(\text { h.c. }) \\
& \left.+\left(b_{\rho}^{\dagger} \cdot b_{\rho}^{\dagger}+b_{\lambda}^{\dagger} \cdot b_{\lambda}^{\dagger}-2 b_{\eta}^{\dagger} \cdot b_{\eta}^{\dagger}\right)(\text { h.c. })\right] .
\end{aligned}
$$

This Hamiltonian is a particular combination of the $U(9)$ and $S O(10)$ dynamical symmetries. For $R^{2}=0$, this Hamiltonian has $U(10) \supset U(9)$ symmetry and corresponds to a nine-dimensional anharmonic oscillator, whereas for $R^{2}=1$ and $\xi_{2}=\xi_{3}=0$ it has $U(10) \supset S O(10)$ symmetry and corresponds to a deformed oscillator. For the general case with $R^{2} \neq 0$ and $\xi_{1}, \xi_{2}, \xi_{3}>0$ the energy spectrum cannot be obtained in closed analytic form. Even though the Hamiltonian of Eq. (14) does not correspond to a dynamical symmetry, an approximate energy formula can still be derived by studying the classical limit of Eq. (14).

The classical limit of the ACM Hamiltonian is defined by the coherent state expectation value

$$
H_{\mathrm{cl}}=\frac{1}{N}\left\langle N ; \vec{\alpha}_{\rho}, \vec{\alpha}_{\lambda}, \vec{\alpha}_{\eta}|: H:| N ; \vec{\alpha}_{\rho}, \vec{\alpha}_{\lambda}, \vec{\alpha}_{\eta}\right\rangle
$$

where the coherent state has the form of a condensate wave function

$$
\left|N ; \vec{\alpha}_{\rho}, \vec{\alpha}_{\lambda}, \vec{\alpha}_{\eta}\right\rangle=\frac{1}{\sqrt{N !}}\left(b_{c}^{\dagger}\right)^{N}|0\rangle
$$

The condensate boson $b_{c}^{\dagger}$ is parametrized in terms of nine complex variables corresponding to the three vector coordinates and their conjugate momenta

$$
b_{c}^{\dagger}=\sqrt{1-\vec{\alpha}_{\rho} \cdot \vec{\alpha}_{\rho}^{*}-\vec{\alpha}_{\lambda} \cdot \vec{\alpha}_{\lambda}^{*}-\vec{\alpha}_{\eta} \cdot \vec{\alpha}_{\eta}^{*}} s^{\dagger}+\vec{\alpha}_{\rho} \cdot \vec{b}_{\rho}^{\dagger}+\vec{\alpha}_{\lambda} \cdot \vec{b}_{\lambda}^{\dagger}+\vec{\alpha}_{\eta} \cdot \vec{b}_{\eta}^{\dagger}
$$

For the geometrical analysis of the ACM Hamiltonian it is convenient to make a transformation to spherical coordinates and momenta $[56,57]$

$$
\alpha_{k, \mu}=\frac{1}{\sqrt{2}} \sum_{\nu} \mathcal{D}_{\mu \nu}^{(1)}\left(\phi_{k}, \theta_{k}, 0\right) \beta_{k, \nu},
$$


with

$$
\left(\begin{array}{c}
\beta_{k, 1} \\
\beta_{k, 0} \\
\beta_{k,-1}
\end{array}\right)=\left(\begin{array}{c}
{\left[-p_{\phi_{k}} / \sin \theta_{k}-i p_{\theta_{k}}\right] / q_{k} \sqrt{2}} \\
q_{k}+i p_{k} \\
{\left[-p_{\phi_{k}} / \sin \theta_{k}+i p_{\theta_{k}}\right] / q_{k} \sqrt{2}}
\end{array}\right),
$$

where $k=\rho, \lambda, \eta$, followed by a change of variables to the hyperspherical coordinates $q, \zeta$ and $\chi$

$$
\begin{aligned}
& q_{\rho}=q \sin \zeta \sin \chi, \\
& q_{\lambda}=q \sin \zeta \cos \chi, \\
& q_{\eta}=q \cos \zeta,
\end{aligned}
$$

and to center-of-mass $\Omega$ and relative angles $\theta_{i j}$ and their conjugate momenta. Here $2 \theta_{\rho \lambda}$ denotes the relative angle between $\vec{\alpha}_{\rho}$ and $\vec{\alpha}_{\lambda}$, and similarly for $2 \theta_{\lambda \eta}$ and $2 \theta_{\eta \rho}$.

The potential energy surface associated with $H_{3, \text { vib }}$ is obtained by setting all momenta equal to zero in the general expression for the classical limit. The equilibrium configuration corresponds to coordinates that have equal length $\left(q_{\rho, 0}=q_{\lambda, 0}=q_{\eta, 0}=q_{0} / \sqrt{3}\right)$

$$
q_{0}=\sqrt{2 R^{2} /\left(1+R^{2}\right)}, \quad \zeta_{0}=\arctan \sqrt{2}, \quad \chi_{0}=\pi / 4,
$$

and are mutually perpendicular

$$
\theta_{\rho \lambda, 0}=\theta_{\lambda \eta, 0}=\theta_{\eta \rho, 0}=\pi / 4 .
$$

In the limit of small oscillations around the equilibrium shape, the intrinsic degrees of freedom decouple and become harmonic. To leading order in $N$ one finds the vibrational energy spectrum of a spherical top with tetrahedral symmetry [58]

$$
E_{3, \mathrm{vib}}=\omega_{1}\left(v_{1}+\frac{1}{2}\right)+\omega_{2}\left(v_{2}+1\right)+\omega_{3}\left(v_{3}+\frac{3}{2}\right) .
$$

The frequencies are related to the $\xi$ coefficients in the vibrational Hamiltonian of Eq. (14)

$$
\omega_{1}=4 N R^{2} \xi_{1}, \quad \omega_{2}=\frac{8 N R^{2}}{1+R^{2}} \xi_{2}, \quad \omega_{3}=\frac{8 N R^{2}}{1+R^{2}} \xi_{3} .
$$

Here $v_{1}$ represents the vibrational quantum number for a symmetric stretching $A_{1}$ vibration, $v_{2}=v_{2 a}+v_{2 b}$ denotes a doubly degenerate $E$ vibration, and $v_{3}=v_{3 a}+v_{3 b}+v_{3 c}$ a three-fold degenerate $F_{2}$ vibration (see Fig. 2). For rigid configurations, $R^{2}=1$, and $\omega_{i}=4 N \xi_{i}$ with $i=1,2,3$.

Next we consider the rotational part of the Hamiltonian

$$
H_{3, \text { rot }}=\kappa_{1} \vec{L} \cdot \vec{L}+\kappa_{2}(\vec{L} \cdot \vec{L}-\vec{I} \cdot \vec{I})^{2},
$$



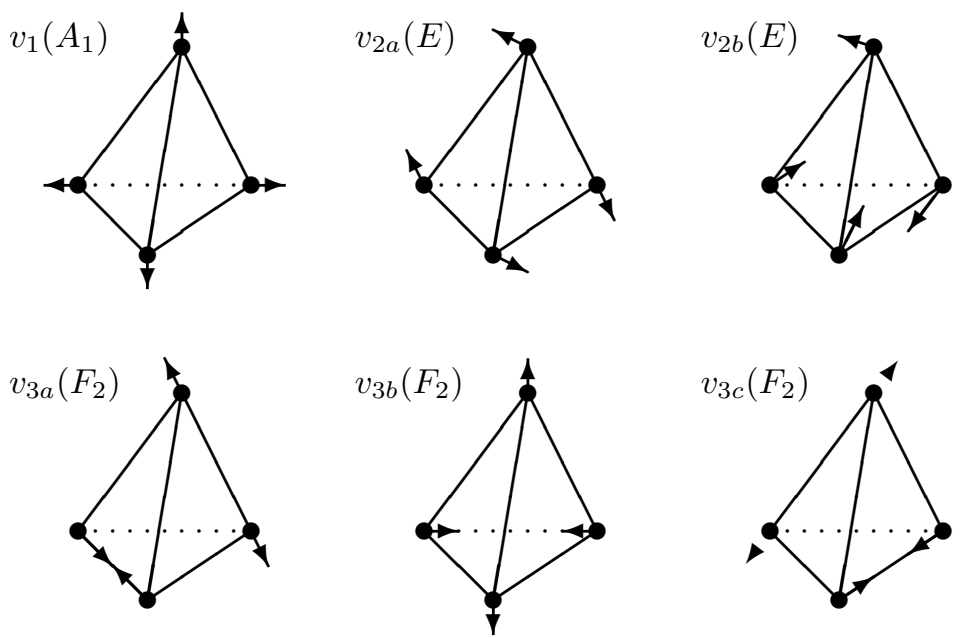

Figure 2: Fundamental vibrations of a tetrahedral configuration (point group $\mathcal{T}_{d}$ ).

where $\vec{L}$ and $\vec{I}$ denote the angular momentum in coordinate space and index space, respectively,

$$
\begin{aligned}
L_{m} & =\sqrt{2} \hat{B}_{m}^{(1)}, & & m=-1,0,1 \\
I_{j} & =\sqrt{3} \hat{K}_{j}^{(0)}, & & j=\rho, \lambda, \eta
\end{aligned}
$$

Whereas the angular momentum $L$ is an exact symmetry of the $\mathcal{T}_{d} \sim S_{4}$ invariant Hamiltonian of Eq. (12), the angular momentum in index space $I$ in general does not commute with the Hamiltonian. Only if $a_{L}=c_{L}$ in Eq. (12) and $\xi_{2}=\xi_{3}$ in Eq. (14), does $I$ become a good quantum number. The rotational excitations of the ground state vibrational band of $H_{3}$ with $\left(v_{1} v_{2} v_{3}\right)=(000)$ are characterized by $L=I$. This property comes from the fact that the operator $\vec{L} \cdot \vec{L}-\vec{I} \cdot \vec{I}$ annihilates the coherent (or intrinisic) state corresponding to the rigid equilibrium shape of Eqs. $(21,22)$. As a consequence, the rotational energies of the ground state band are given by $\kappa_{1} L(L+1)$.

$$
E_{3, \text { rot }}=\kappa_{1} L(L+1) .
$$

Fig. 3 shows the structure of the rotational excitations of the ground state band $\left(v_{1} v_{2} v_{3}\right)=(000)$. The rotational levels are doubled because of inversion doubling: for each value of the angular momentum $L$, one has doublets of states with $\left(A_{1}, A_{2}\right),(E, E)$ and $\left(F_{2}, F_{1}\right)$, in agreement with the classification of 


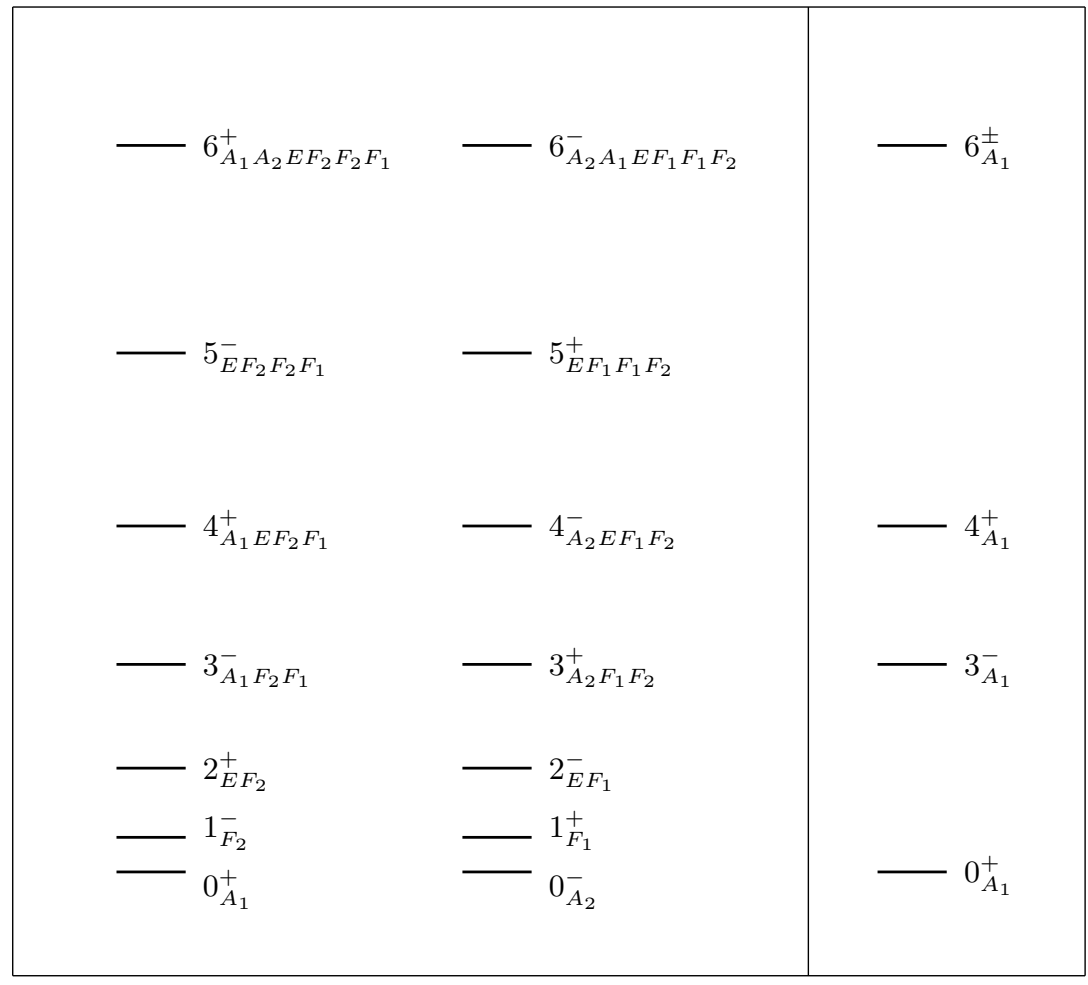

Figure 3: Schematic spectrum of the rotational states of the ground stand vibrational band with $\left(v_{1} v_{2} v_{3}\right)=(000)$. All states have $L=I$ and are labeled by $L_{t}^{P}$. On the right-hand side we show the symmetric states with $t=A_{1}$.

rotational levels of a spherical top with tetrahedral symmetry $[58,59]$. For identical bosons, as is the case for a cluster of four $\alpha$-particles, the allowed rotational-vibrational states are the symmetric ones with $t=A_{1}$, and therefore the states of the ground state band have angular momentum and parity $L^{P}=$ $0^{+}, 3^{-}, 4^{+}, 6^{ \pm}, \ldots$, as shown in the right-hand side of Fig. 3. A similar analysis can be done for the rotational bands built on the (100) $A_{1},(010) E$ and $(001) F_{2}$ vibrations. For the $A_{1}$ vibration the values of angular momentum and parity are the same as for the ground state band $L^{P}=0^{+}, 3^{-}, 4^{+}, 6^{ \pm}, \ldots$ For the doubly degenerate $E$ vibration they are $L^{P}=2^{ \pm}, 4^{ \pm}, 5^{ \pm}, 6^{ \pm}, \ldots$, while for the triply degenerate $F_{2}$ vibration they are $L^{P}=1^{-}, 2^{+}, 3^{ \pm}, 4^{ \pm}, 5^{-, \pm}, 6^{+, \pm} \ldots$ The situation is summarized in Fig. 4 which shows the expected spectrum of a spherical top with tetrahedral symmetry and $\omega_{1}=\omega_{2}=\omega_{3}$.

\section{Transition probabilities}

In order to calculate transition form factors and transition probabilities in the algebraic cluster model one has to express the transition operators in terms 


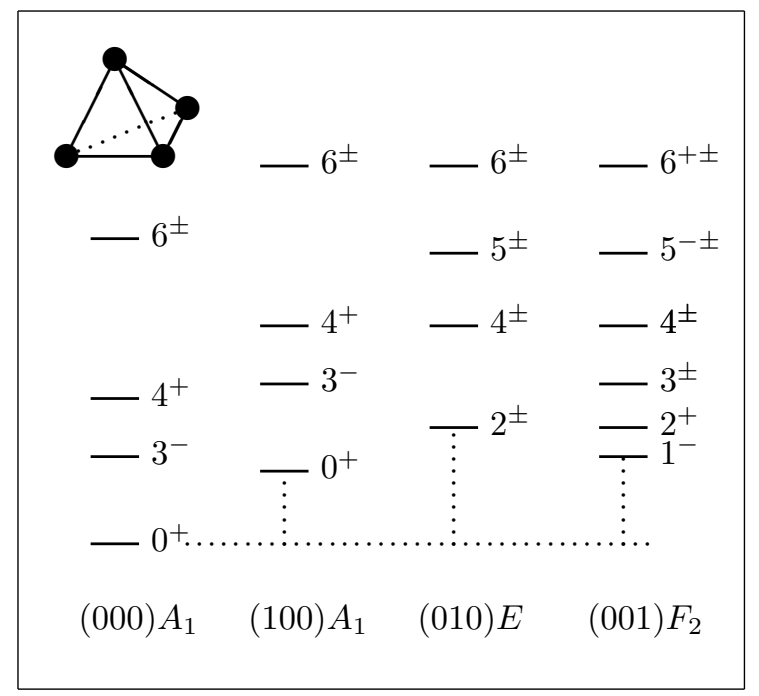

Figure 4: Schematic spectrum of a spherical top with tetrahedral symmetry and $\omega_{1}=\omega_{2}=\omega_{3}$. The rotational bands are labeled by $\left(v_{1} v_{2} v_{3}\right)$ (bottom). All states are symmetric under $S_{4} \sim \mathcal{T}_{d}$.

of the algebraic operators. The transition form factors are the matrix elements of $\sum_{i=1}^{4} \exp \left(i \vec{q} \cdot \vec{r}_{i}\right)$ where $\vec{q}$ is the momentum transfer. Choosing the $z$-axis along the direction of the momentum transfer and using the fact that the four particles are identical, it is sufficient to consider the matrix elements of $\exp \left(i q r_{4 z}\right)$. After converting to Jacobi coordinates and integrating over the center-of-mass coordinate one has $\exp \left(-i q \sqrt{3 / 4} \eta_{z}\right)$. The matrix elements of this operator can be obtained algebraically by making the replacement

$$
\sqrt{3 / 4} \eta_{z} \rightarrow \beta \hat{D}_{\eta, z} / X_{D}
$$

where $\beta$ represents the scale of the coordinate and $X_{D}$ is given by the reduced matrix element of the dipole operator. The replacement in Eq. (28) comes from the fact that in the large $N$ limit, the dipole operators $\hat{D}_{\rho}, \hat{D}_{\lambda}$ and $\hat{D}_{\eta}$ of Eq. (11) correspond to the three Jacobi coordinates $\vec{\rho}, \vec{\lambda}$ and $\vec{\eta}[56]$.

In summary, the transition form factors can be expressed in the ACM as

$$
\mathcal{F}_{M}(i \rightarrow f ; q)=\left\langle\gamma_{f}, L_{f}, M|\hat{T}(\epsilon)| \gamma_{i}, L_{i}, M\right\rangle
$$

with

$$
\hat{T}(\epsilon)=e^{i \epsilon \hat{D}_{\eta, z}}=e^{-i q \beta \hat{D}_{\eta, z} / X_{D}} .
$$

The transition probabilities $B(E L)$ can be extracted from the form factors in the long wavelength limit

$$
B(E L ; i \rightarrow f)=(Z e)^{2} \frac{[(2 L+1) ! !]^{2}}{4 \pi\left(2 L_{i}+1\right)} \lim _{q \rightarrow 0} \sum_{M} \frac{\left|\mathcal{F}_{M}(i \rightarrow f ; q)\right|^{2}}{q^{2 L}}
$$


where $Z e$ is the total electric charge of the cluster. In the three special cases of the harmonic oscillator, or $U(9)$ limit, the deformed oscillator, or $S O(10)$ limit, and the spherical top with $\mathcal{T}_{d}$ symmetry, the matrix elements of $\hat{T}$ can be obtained analytically and are given in the subsections below.

For the general ACM Hamiltonian of Eq. (12), the matrix elements cannot be obtained in closed analytic form, but have to be calculated numerically. Hereto, a computer program has been developed [60], in which the form factors are obtained exactly by using the symmetry properties of the transition operator of Eq. (30).

When a dynamic symmetry occurs the matrix elements of the transition operators can be obtained in explicit analytic form. The general procedure to derive the transition form factors in the harmonic oscillator and deformed oscillator limits was discussed in [61, 62].

In the $U(9)$ limit of the ACM the elastic form factor can be derived as

$$
\mathcal{F}\left(0^{+} \rightarrow 0^{+} ; q\right)=(\cos \epsilon)^{N} \rightarrow e^{-q^{2} \beta^{2} / 6},
$$

with $\epsilon=-q \beta / X_{D}$ and $X_{D}=\sqrt{3 N}$. In the large $N$ limit, the elastic form factor exhibits an exponential fall-off with momentum transfer.

In the $S O(10)$ limit the elastic form factor is given in terms of a Gegenbauer polynomial

$$
\mathcal{F}\left(0^{+} \rightarrow 0^{+} ; q\right)=\frac{7 ! N !}{(N+7) !} C_{N}^{(4)}(\cos \epsilon) \rightarrow \frac{7 ! ! j_{3}(q \beta \sqrt{3})}{(q \beta \sqrt{3})^{3}} .
$$

with $\epsilon=-q \beta / X_{D}$ and $X_{D}=\sqrt{N(N+8) / 3}$. In the large $N$ limit, the elastic form factor is proportional to a spherical Bessel function.

For the spherical top with $\mathcal{T}_{d}$ symmetry, the form factors can only be obtained in closed form in the large $N$ limit using a technique introduced in [63] and subsequently exploited in [45]. The normalization factor $X_{D}$ which appears in the algebraic transition operator of Eq. (30), is here $X_{D}=2 N R /\left(1+R^{2}\right) \sqrt{3}$. The elastic form factor can be obtained as

$$
\begin{aligned}
\mathcal{F}\left(0^{+} \rightarrow 0^{+} ; q\right) & \rightarrow \frac{1}{4 \pi} \int d \Omega\left\langle N, c\left|\mathcal{R}^{-1}(\Omega) \hat{T}(\epsilon) \mathcal{R}(\Omega)\right| N, c\right\rangle \\
& =\frac{1}{2} \int d \cos \theta e^{-i q \beta \cos \theta}=j_{0}(q \beta) .
\end{aligned}
$$

In Eq. (34), $|N, c\rangle$ is the condensate corresponding to a tetrahedral equilibrium configuration

$$
\begin{aligned}
|N, c\rangle & =\frac{1}{\sqrt{N !}}\left(b_{c}^{\dagger}\right)^{N}|0\rangle, \\
b_{c}^{\dagger} & =\frac{1}{\sqrt{1+R^{2}}}\left(s^{\dagger}+\frac{R}{\sqrt{3}}\left(b_{\rho x}^{\dagger}+b_{\lambda y}^{\dagger}+b_{\eta z}^{\dagger}\right)\right) .
\end{aligned}
$$

In general, for transitions along the ground state band (000) $A_{1}$ the transition form factors are given in terms of a spherical Bessel function

$$
\mathcal{F}\left(0^{+} \rightarrow L^{P} ; q\right) \rightarrow c_{L} j_{L}(q \beta)
$$


Table 1: Transition form factors for the ground state band of the spherical top.

\begin{tabular}{cc}
\hline$L^{P}$ & $\left|\mathcal{F}\left(0^{+} \rightarrow L^{P} ; q\right)\right|$ \\
\hline $0^{+}$ & $j_{0}(q \beta)$ \\
$3^{-}$ & $\sqrt{\frac{35}{9}} j_{3}(q \beta)$ \\
$4^{+}$ & $\sqrt{\frac{7}{3}} j_{4}(q \beta)$ \\
$6^{+}$ & $\sqrt{\frac{416}{81}} j_{6}(q \beta)$ \\
\hline
\end{tabular}

with

$$
c_{L}^{2}=\frac{2 L+1}{16}\left[4+12 P_{L}\left(-\frac{1}{3}\right)\right] .
$$

The coefficients $c_{1}^{2}, c_{2}^{2}$ and $c_{5}^{2}$ vanish as a consequence of the tetrahedral symmetry. Some values which are relevant to the lowest states are $c_{0}^{2}=1, c_{3}^{2}=35 / 9$, $c_{4}^{2}=7 / 3$ and $c_{6}^{2}=416 / 81$.

The transition probabilities $B(E L)$ along the ground state band can be extracted from the form factors in the long wavelength limit according to Eq. (31). The result is given by a simple formula

$$
B\left(E L ; 0^{+} \rightarrow L^{P}\right)=\left(\frac{Z e \beta^{L}}{4}\right)^{2} \frac{2 L+1}{4 \pi}\left[4+12 P_{L}\left(-\frac{1}{3}\right)\right]
$$

with $P=(-1)^{L}$. Explicit expressions are

$$
\begin{aligned}
B\left(E 3 ; 0^{+} \rightarrow 3^{-}\right) & =(Z e)^{2} \frac{7}{4 \pi} \frac{5}{9} \beta^{6}, \\
B\left(E 4 ; 0^{+} \rightarrow 4^{+}\right) & =(Z e)^{2} \frac{9}{4 \pi} \frac{7}{27} \beta^{8}, \\
B\left(E 6 ; 0^{+} \rightarrow 6^{+}\right) & =(Z e)^{2} \frac{13}{4 \pi} \frac{32}{81} \beta^{12} .
\end{aligned}
$$

Form factors and $B(E L)$ values only depend on the parameter $\beta$, the distance of each particle from the center of mass of the tetrahedral configuration, and on the $\mathcal{T}_{d}$ symmetry which gives the coefficients $c_{L}$. The analytic results given in this section provide a set of closed expressions which can be compared with experiment.

All results for form factors given in Eqs. (32-34) and Table 1 are for point-like constituent particles with a charge distribution

$$
\rho(\vec{r})=\frac{Z e}{4} \sum_{i=1}^{4} \delta\left(\vec{r}-\vec{r}_{i}\right),
$$


where $Z e$ is the total electric charge. In cases in which the constituent particles are composite particles and thus have an intrinsic form factor they must be modified. Assuming a Gaussian form,

$$
\rho(\vec{r})=\frac{Z e}{4}\left(\frac{\alpha}{\pi}\right)^{3 / 2} \sum_{i=1}^{4} \exp \left[-\alpha\left(\vec{r}-\vec{r}_{i}\right)^{2}\right]
$$

all form factors are multiplied by an exponential factor $\exp \left(-q^{2} / 4 \alpha\right)$. The charge radius can be obtained from the slope of the elastic form factor in the origin

$$
\left\langle r^{2}\right\rangle^{1 / 2}=\left[-\left.6 \frac{d \mathcal{F}\left(0^{+} \rightarrow 0^{+} ; q\right)}{d q^{2}}\right|_{q=0}\right]^{1 / 2}=\sqrt{\frac{3}{2 \alpha}+\beta^{2}} .
$$

The above formula for the charge radius is valid for all three cases discussed in this section: the harmonic oscillator, the deformed oscillator and the spherical top.

It is of great interest to determine the charge distribution in the case in which one has composite particles at the vertices of a tetrahedron. This charge distribution is given by the expansion of Eq. (41) into multipoles

$$
\rho(\vec{r})=\frac{Z e}{4}\left(\frac{\alpha}{\pi}\right)^{3 / 2} e^{-\alpha\left(r^{2}+\beta^{2}\right)} \sum_{\lambda=0}^{\infty}(2 \lambda+1) i_{\lambda}(2 \alpha \beta r) \sum_{i=1}^{4} P_{\lambda}\left(\cos \gamma_{i}\right)
$$

with

$$
\cos \gamma_{i}=\cos \theta \cos \theta_{i}+\sin \theta \sin \theta_{i} \cos \left(\phi-\phi_{i}\right) .
$$

Here we have used spherical coordinates $\vec{r}=(r, \theta, \phi)$ and $\vec{r}_{i}=\left(\beta, \theta_{i}, \phi_{i}\right)$. The angles $\theta_{i}$ and $\phi_{i}$ denote the location of the four particles, and $i_{\lambda}(x)=j_{\lambda}(i x) / i^{\lambda}$ is the modified spherical Bessel function. This formula is valid for all cases in which the distance from the origin is the same $\left|\vec{r}_{i}\right|=\beta$ for all constituents. In the case of $n$ constituents, the charge of the constituent in Eq. (43) is changed from $Z e / 4$ to $Z e / n$, and the sum is over all constituents from 1 to $n$.

\section{The nucleus ${ }^{16} \mathrm{O}$}

The nucleus ${ }^{16} \mathrm{O}$ has been the subject of many investigations. Within the framework of the nuclear shell model detailed studies were made by Zuker et al. [64] in terms of $1 \mathrm{p}-1 \mathrm{~h}$ and by Brown and Green [65] and Feshbach and Iachello [66] in terms of multiparticle-multihole configurations. However, as early as 1954, Dennison suggested that its spectrum could be understood in terms of an $\alpha$-particle model with $\mathcal{T}_{d}$ symmetry [3]. This idea was adopted by Kameny [4], Brink [5, 6], and especially by Robson [7, 8, 67, 68], who in a series of papers developed the model in further detail. Other possible configurations were also studied, in particular $\alpha+{ }^{12} \mathrm{C}$ [69]. 


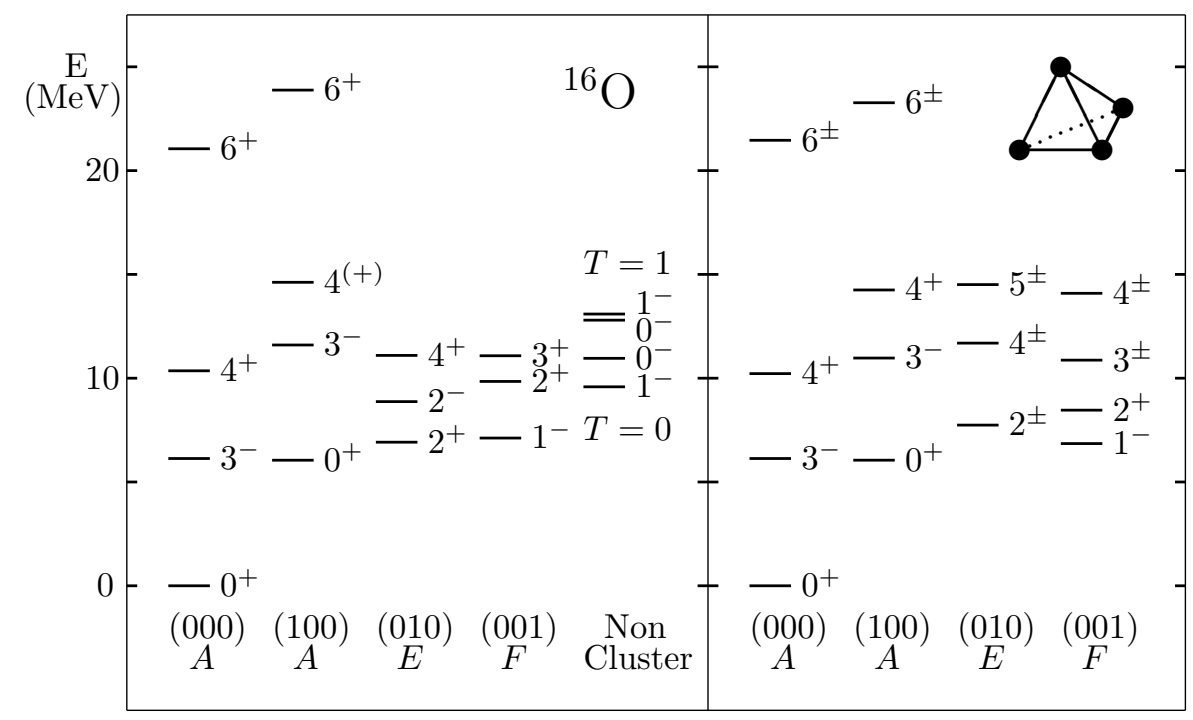

Figure 5: Comparison between the observed spectrum of ${ }^{16} \mathrm{O}$ (left) and the theoretical spectrum (right). The levels are organized in columns corresponding to the ground state band and the three vibrational bands with $A, E$ and $F$ symmetry of a spherical top with tetrahedral symmetry. The last column shows the lowest non-cluste $\mathrm{r}$ levels.

In very recent years, ${ }^{16} \mathrm{O}$ has been again the subject of many investigations, both within the framework of the no-core shell model [70] and $a b$ initio lattice calculations [71]. The latter shows evidence for tetrahedral symmetry in the ground state of ${ }^{16} \mathrm{O}$. Another important development has been the suggestion that some of the excited $0^{+}$states may have a large $\alpha$-condensate fraction and/or $\alpha+{ }^{12} \mathrm{C}$ cluster structure [72, 73].

In this article, we analyze the available data in the terms of the spherical top limit of the $U(10)$ algebraic cluster model with $\mathcal{T}_{d}$ symmetry.

\subsection{Energies}

There are 340 levels known in ${ }^{16} \mathrm{O}$ in the energy range $0-35 \mathrm{MeV}$ [74]. We have first analyzed these levels with the simple formula for a rigid spherical top with tetrahedral symmetry

$$
E\left(v_{1}, v_{2}, v_{3}, L\right)=E_{0}+\omega_{1} v_{1}+\omega_{2} v_{2}+\omega_{3} v_{3}+B_{[v]} L(L+1)
$$

where the zero-point energy is given by $E_{0}=\frac{1}{2} \omega_{1}+\omega_{2}+\frac{3}{2} \omega_{3}$. By taking $\omega_{1}=$ $\omega_{2}=\omega_{3}=6.05 \mathrm{MeV}, B_{000 A}=0.511 \mathrm{MeV}, B_{100 A}=0.410 \mathrm{MeV}, B_{010 E}=0.282$ $\mathrm{MeV}$ and $B_{001 F}=0.402 \mathrm{MeV}$, we are able to obtain an excellent description of the low-lying spectrum of ${ }^{16} \mathrm{O}$, a portion of which is shown in Fig. 5. Our classification includes 17 states firmly assigned to $\mathcal{T}_{d}$ symmetry and another 11 tentatively assigned (in parentheses) covering the range from 0 to $29 \mathrm{MeV}$, 
Table 2: Classification of levels in ${ }^{16} \mathrm{O}$ in terms of $\mathcal{T}_{d}$ symmetry for the ground state band and single vibrational excitations.

\begin{tabular}{|c|c|c|c|}
\hline$\left(v_{1}, v_{2}, v_{3}\right)$ & $L^{P}$ & $E_{\mathrm{th}}(\mathrm{MeV})$ & $E_{\exp }(\mathrm{MeV})$ \\
\hline \multirow[t]{7}{*}{$(000) A_{1}$} & $0^{+}$ & 0.00 & 0.000 \\
\hline & $3^{-}$ & 6.13 & 6.130 \\
\hline & $4^{+}$ & 10.22 & 10.356 \\
\hline & $6^{+}$ & 21.46 & 21.052 \\
\hline & $6^{-}$ & 21.46 & \\
\hline & $7^{-}$ & 28.62 & $(28.2)$ \\
\hline & $8^{+}$ & 36.79 & \\
\hline \multirow[t]{7}{*}{$(100) A_{1}$} & $0^{+}$ & 6.05 & 6.049 \\
\hline & $3^{-}$ & 10.97 & 11.600 \\
\hline & $4^{+}$ & 14.25 & 14.620 \\
\hline & $6^{+}$ & 23.27 & 23.880 \\
\hline & $6^{-}$ & 23.27 & \\
\hline & $7^{-}$ & 29.01 & $(29.0)$ \\
\hline & $8^{+}$ & 35.57 & \\
\hline \multirow[t]{8}{*}{$(010) E$} & $2^{+}$ & 7.74 & 6.917 \\
\hline & $2^{-}$ & 7.74 & 8.872 \\
\hline & $4^{+}$ & 11.69 & 11.097 \\
\hline & $4^{-}$ & 11.69 & \\
\hline & $5^{+}$ & 14.51 & 14.399 \\
\hline & $5^{-}$ & 14.51 & 14.660 \\
\hline & $6^{+}$ & 17.89 & $(16.275)$ \\
\hline & $6^{-}$ & 17.89 & \\
\hline \multirow[t]{10}{*}{$(001) F_{2}$} & $1^{-}$ & 6.85 & 7.117 \\
\hline & $2^{+}$ & 8.46 & 9.844 \\
\hline & $3^{+}$ & 10.87 & 11.080 \\
\hline & $3^{-}$ & 10.87 & \\
\hline & $4^{+}$ & 14.09 & $(13.869)$ \\
\hline & $4^{-}$ & 14.09 & $(14.302)$ \\
\hline & $5^{ \pm}$ & 18.11 & \\
\hline & $5^{-}$ & 18.11 & \\
\hline & $6^{ \pm}$ & 22.93 & \\
\hline & $6^{+}$ & 22.93 & $(23.0)$ \\
\hline
\end{tabular}

as shown in Tables 2 and 3 . We note that because of the multiplication rule $E \otimes F_{2}=F_{1} \oplus F_{2}$ the vibration (011) contains $F_{1}$.

The rotational ground state band with angular momenta $L^{P}=0^{+}, 3^{-}, 4^{+}$, $6^{+}$has been observed with moment of inertia such that $B_{000 A}=0.511 \mathrm{MeV}$. The spherical top predicts a $L^{P}=6^{ \pm}$doublet as a consequence of the tetrahedral symmetry. The $6^{-}$state has not been identified yet. It appears that all three vibrations (100) $A_{1},(010) E$ and $(001) F_{2}$ have been identified with comparable energy $\omega_{1}=\omega_{2}=\omega_{3}=6.05 \mathrm{MeV}$ as one would expect from Eq. (14) with 
Table 3: Classification of levels in ${ }^{16} \mathrm{O}$ in terms of $\mathcal{T}_{d}$ symmetry for double vibrational excitations.

\begin{tabular}{clcc}
\hline$\left(v_{1}, v_{2}, v_{3}\right)$ & $L^{P}$ & $E_{\mathrm{th}}(\mathrm{MeV})$ & $E_{\exp }(\mathrm{MeV})$ \\
\hline$(200) A_{1}$ & $0^{+}$ & 12.10 & 12.049 \\
& $3^{-}$ & & \\
\hline$(110) E$ & $2^{+}$ & & $(11.520)$ \\
& $2^{-}$ & & \\
\hline$(101) F_{2}$ & $1^{-}$ & & $(12.440)$ \\
& $2^{+}$ & & $(13.020)$ \\
\hline$(020) A_{1}$ & $0^{+}$ & 12.10 & \\
$(020) E$ & $2^{-}$ & & \\
& $2^{+}$ & & $(11.260)$ \\
\hline$(002) A_{1}$ & $0^{+}$ & 12.10 & $(13.664)$ \\
\hline$(011) F_{1}$ & $1^{+}$ & & \\
\hline
\end{tabular}

$\xi_{1}=\xi_{2}=\xi_{3}$. Members of the rotational bands have also been observed. With the present assignments of $L^{P}=0^{+}, 3^{-}, 4^{+}, 6^{+}$states of the the breathing mode (100) $A$, this vibrational band has a moment of inertia very similar to that of the ground state band. This band is similar in nature to the band built on the Hoyle state in ${ }^{12} \mathrm{C}$ which was recently measured experimentally $[18,19,20,21,22]$ and reviewed in [15]. The moments of inertia of the (100) $A_{1},(010) E$ and $(001) F_{2}$ bands are larger (smaller $B$ values) than that of the ground state band due to their nature (breathing and bending vibrations). The moments of inertia of the $A_{1}$ and $F_{2}$ vibrations have almost the same value (and somewhat larger than that of the ground state band), whereas the moment of inertia of the $E$ vibration has almost double the value of that of the ground state band. The situation is summarized in Fig. 6. It appears also that some members of the double vibrations (200) $A_{1},(110) E,(101) F_{2},(002) A_{1}$ and $(011) F_{1}$ can be identified. The vibrations are nearly harmonic.

A characteristic feature of the spectrum of a spherical top with tetrahedral symmetry is the occurrence of parity doublets both in the ground state band and in the vibrational excitations (see Fig. 4). In the ground state band and the (100) $A_{1}$ breathing vibration one expects an additional $L^{P}=6^{-}$state, in the $(010) E$ vibration an extra $4^{-}$state and in the $(001) F_{2}$ vibration a $3^{-}$state is missing (see Table 2).

The experimental spectrum is very similar to that of a spherical top with tetrahedral symmetry. Nevertheless there are some perturbations which can be described by adding higher order terms to the Hamiltonian, and consequently to the energy formula of Eq. (45). Simple perturbations are

(i) vibrational anharmonicities which contribute as $x_{i j} v_{i} v_{j}$, 


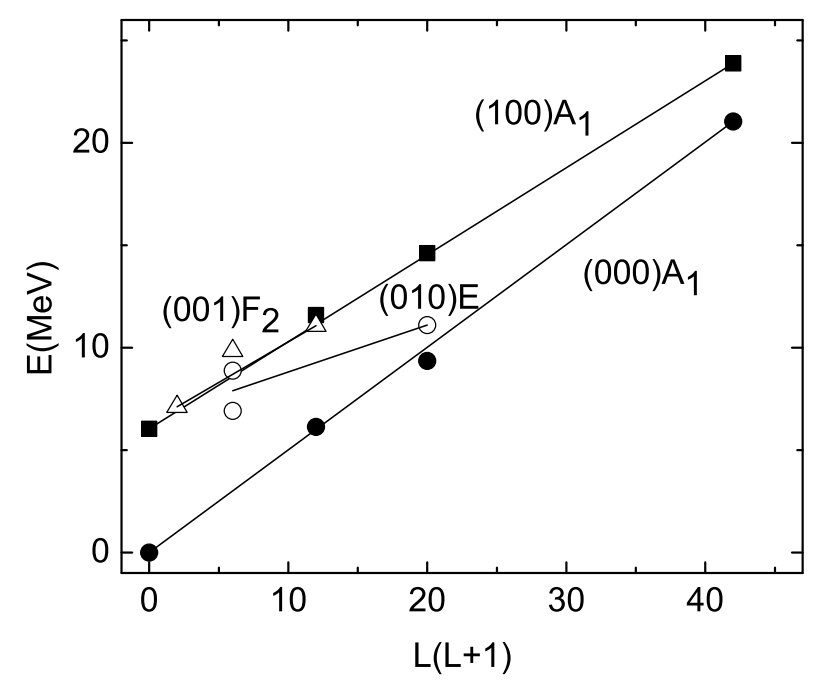

Figure 6: The excitation energies of cluster states in ${ }^{16} \mathrm{O}$ plotted as a function of $L(L+1)$ : closed circles for the ground state band $\left(0^{+}, 3^{-}, 4^{+}, 6^{+}\right)$, closed squares for the $A_{1}$ vibration $\left(0^{+}, 3^{-}, 4^{+}, 6^{+}\right)$, open circles for the $E$ vibration $\left(2^{+}, 2^{-}, 4^{+}\right)$and open triangles for the $F_{2}$ vibration $\left(1^{-}, 2^{+}, 3^{+}\right)[32]$.

(ii) centrifugal stretching which leads to corrections of the form $D_{[v]} L^{2}(L+1)^{2}$ (iii) Coriolis coupling. This only affects $F$ representations and causes a splitting of the triply degenerate vibration into three pieces

$$
\begin{aligned}
F^{(+)} & =B_{[v]} L(L+1)+2 B_{[v]} \zeta(L+1), \\
F^{(0)} & =B_{[v]} L(L+1), \\
F^{(-)} & =B_{[v]} L(L+1)-2 B_{[v]} \zeta(L+1) .
\end{aligned}
$$

According to an estimate by Dennison, the coefficient $\zeta=1 / 2$ in ${ }^{16} \mathrm{O}[75]$.

(iv) Rotation-vibration interaction. The most notable consequence is a signature splitting

$$
\Delta=\left[\frac{1+(-1)^{P}}{2}\right] \eta_{[v]},
$$

which splits the positive parity from the negative parity states with the same angular momentum, for example $2^{+}$and $2^{-}$. This effect is particularly important for the $E$ vibration.

However, deviations from the simple energy formula of Eq. (45) appear to be small with the only exception of the $2^{+} / 2^{-}$splitting in the $E$ vibration which corresponds to $\Delta=1.955 \mathrm{MeV}$, and we therefore do not discuss higher order terms further. Our assignments are in part different from those of Robson [8] who introduced a large centrifugal stretching and other perturbations. Within 
Table 4: Classification of levels in ${ }^{16} \mathrm{O}$ in terms of specific shell-model configurations.

\begin{tabular}{ccc}
\hline Classification & $L^{P}$ & $E_{\text {exp }}(\mathrm{MeV})$ \\
\hline 1p-1h $p_{1 / 2}^{-1} s_{1 / 2}$ & $1^{-} T=0$ & 9.585 \\
& $0^{-} T=0$ & 10.957 \\
\hline 1p-1h $p_{1 / 2}^{-1} d_{5 / 2}$ & $2^{-} T=0$ & 12.530 \\
& $3^{-} T=0$ & 13.129 \\
\hline 1p-1h $p_{1 / 2}^{-1} s_{1 / 2}$ & $0^{-} T=1$ & 12.796 \\
& $1^{-} T=1$ & 13.090 \\
\hline 1p-1h $p_{1 / 2}^{-1} d_{5 / 2}$ & $2^{-} T=1$ & 12.968 \\
& $3^{-} T=1$ & 13.259 \\
\hline
\end{tabular}

the framework of the ACM, perturbations can be studied by diagonalizing the Hamiltonian for finite $N$ and $R^{2} \neq 1$.

Our discussion of the spectrum of ${ }^{16} \mathrm{O}$ has been so far assuming no internal excitation of the $\alpha$-particle. However, in addition to the collective cluster excitations we expect also single-particle (shell model) excitations. We can identify uniquely non-cluster states by their isotopic spin nature $(T=1)$ and for $T=0$ by the fact that some spin-parity states are not allowed by the symmetry of the $4 \alpha$ configuration. Specifically, no $0^{-}$state can be formed in states with $v=v_{1}+v_{2}+v_{3}<3$. We are therefore able to identify non-cluster states in the low-lying spectrum as shown in Table 4. Some of the non-cluster states are shown in Fig. 5, to emphasize the fact that all observed states below $9.58 \mathrm{MeV}$ are cluster states. In a shell-model language, cluster states are multiparticlemultihole excitations $[65,66]$.

\subsection{Form factors and electromagnetic transition rates}

Form factors for electron scattering on ${ }^{16} \mathrm{O}$ were measured long ago [76][81]. For transitions along the ground state band the theoretical form factors for the spherical top are given in Table 1. These expressions are only valid in the large $N$ limit. In this section, the form factors are calculated numerically for $N=10$ and $R^{2}=1$. In addition, the form factors are multiplied by an exponential factor $\exp \left(-q^{2} / 4 \alpha\right)$ for an extended distribution. The coefficient $\beta$ in Eq. (30) is determined from the first minimum in the elastic form factor [76] to be $\beta=2.071 \mathrm{fm}$, and subsequently the coefficient $\alpha$ is determined from the charge radius of ${ }^{16} \mathrm{O}$ [74] to be $\alpha=0.605 \mathrm{fm}^{-2}$. If the $\alpha$-particle is not affected by the presence of the others, the coefficient $\alpha$ should be the same as for free $\alpha$-particles. The fitted value is slightly different from the free value $\alpha=0.549$ $\mathrm{fm}^{-2}[82,83,84,85]$ indicating a polarization of the $\alpha$-particle in the medium. A comparison between experimental and calculated form factors in the ground state band is given in Fig. 7. In the figure we have combined the experimental data for the excitation of the $3_{1}^{-}$state (red) $[78,80]$ with those of the unresolved doublet of the $3_{1}^{-}$and $0_{2}^{+}$states at $6.1 \mathrm{MeV}$ (blue) [77, 81]. 

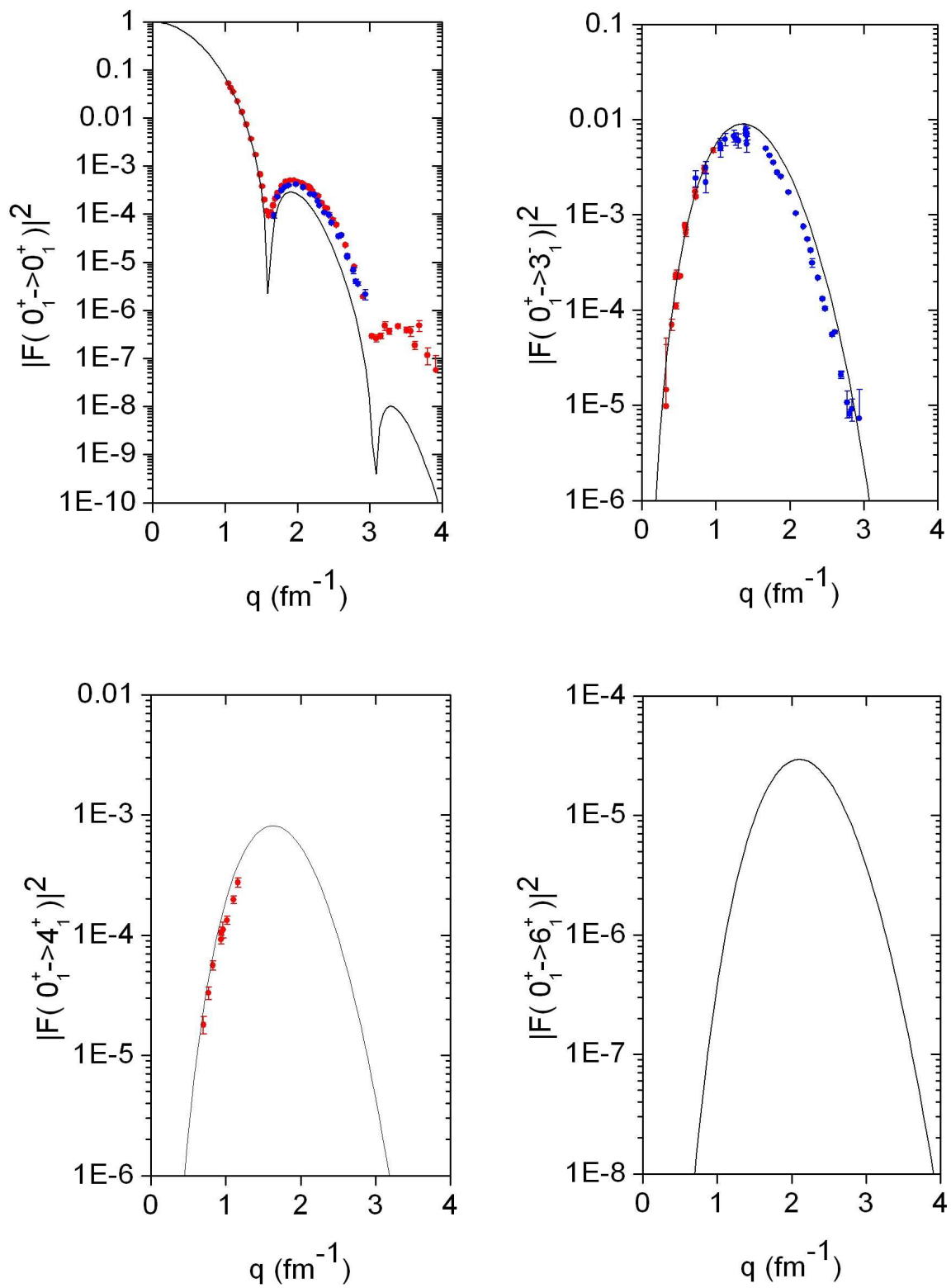

Figure 7: Comparison between the experimental form factors $\left|\mathcal{F}\left(0_{1}^{+} \rightarrow L_{i}^{P}\right)\right|^{2}$ of ${ }^{16} \mathrm{O}$ for the final states with $L_{i}^{P}=0_{1}^{+}, 3_{1}^{-}, 4_{1}^{+}$and $6_{1}^{+}$and those obtained for the spherical top with $N=10$ and $R^{2}=1.0$. The experimental data are taken from Refs. [76, 77, 78, 79, 80, 81]. 
Table 5: Comparison of theoretical and experimental $B(E L)$ values in $\mathrm{e}^{2} \mathrm{fm}^{2 L}$ and $E_{\gamma}$ values in $\mathrm{keV}$, along the ground state band. The theoretical $B(E L)$ values are obtained in the long wavelength limit according to Eq. (31). The $E_{\gamma}$ values are calculated from $E=0.511 L(L+1)$ $\mathrm{MeV}$. The experimental values are taken from [74].

\begin{tabular}{cccccc}
\hline$B\left(E L ; L^{P} \rightarrow 0^{+}\right)$ & Th & $\operatorname{Exp}$ & $E_{\gamma}\left(L^{P}\right)$ & Th & Exp \\
\hline$B\left(E 3 ; 3_{1}^{-} \rightarrow 0_{1}^{+}\right)$ & 215 & $205 \pm 11$ & $E_{\gamma}\left(3_{1}^{-}\right)$ & 6132 & 6130 \\
$B\left(E 4 ; 4_{1}^{+} \rightarrow 0_{1}^{+}\right)$ & 425 & $378 \pm 133$ & $E_{\gamma}\left(4_{1}^{+}\right)$ & 10220 & 10356 \\
$B\left(E 6 ; 6_{1}^{+} \rightarrow 0_{1}^{+}\right)$ & 9626 & & $E_{\gamma}\left(6_{1}^{+}\right)$ & 21462 & 21052 \\
\hline$\left\langle r^{2}\right\rangle^{1 / 2}$ & 2.710 & $2.710 \pm 0.015 \mathrm{fm}$ & & & \\
\hline
\end{tabular}

Electromagnetic transition rates along the ground state band can be obtained in the long wavelength limit of the form factors of Fig. 7 according to Eq. (31). In Table 5, the $B(E L)$ values are compared with experiment. The agreement is excellent and establishes the ground state band as a representation of the tetrahedral group $\mathcal{T}_{d}$ with $A_{1}$ symmetry. Finally, we note that the values presented in [32] are slightly different since those correspond to the analytic results in the large $N$ limit of Eq. (38) with $\beta=2.0 \mathrm{fm}$.

In the ACM, form factors and electromagnetic transition rates from the ground state to the vibrational states are reduced by a factor of $1 / N$ with respect to the rotational excitations of the ground state band. In Fig. 8 we show the results for transitions to the bandheads of the $A_{1}, E$ and $F_{2}$ vibrations.

The corresponding electromagnetic transition rates can be obtained from the form factors in the long wavelength limit according to Eq. (31). In Table 6 they are compared with experiment for the same values of $R^{2}$. The $B\left(E 1 ; 1_{1}^{-} \rightarrow 0_{1}^{+}\right)$ value vanishes due to the tetrahedral symmetry: the initial and final states are symmetric $A_{1}$ whereas the dipole operator has symmetry $F_{2}$. The experimental value is rather small, in agreement with the $\mathcal{T}_{d}$ symmetry. The calculated monopole matrix element is a factor of 6 smaller than the experimental one, indicating that either the transition operator is insufficient to describe transitions from the ground state to the vibrations or that the nature of the vibrations is somewhat different from the assumed one. However, since the shape of the form factor is correctly given by the vibrational behavior and, as shown in Table 2, one can identify a rotational band built on top of the state, we suggest that the assignment of the $0_{2}^{+}$state as the bandhead of the $A_{1}$ vibration is correct, but that to obtain a better agreement one needs higher order terms in the transition operator [86].

Form factors and transition rates among excited states are in general more difficult to calculate. The $E 2$ quadrupole transitions can be calculated numerically. The results are given in Table 7 . The agreement here is poor, especifically for the decays $2_{1}^{+} \rightarrow 0_{2}^{+}$and $4_{1}^{+} \rightarrow 2_{1}^{+}$. With the assignment of $4_{1}^{+}$as a member of the ground state rotational band, the $B\left(E 2 ; 4_{1}^{+} \rightarrow 2_{1}^{+}\right)$value vanishes due to a selection rule for the index spin. All members of the ground state band have $I=L$, so the index spin of the initial state is $I=4$, whereas the $2_{1}^{+}$state has 

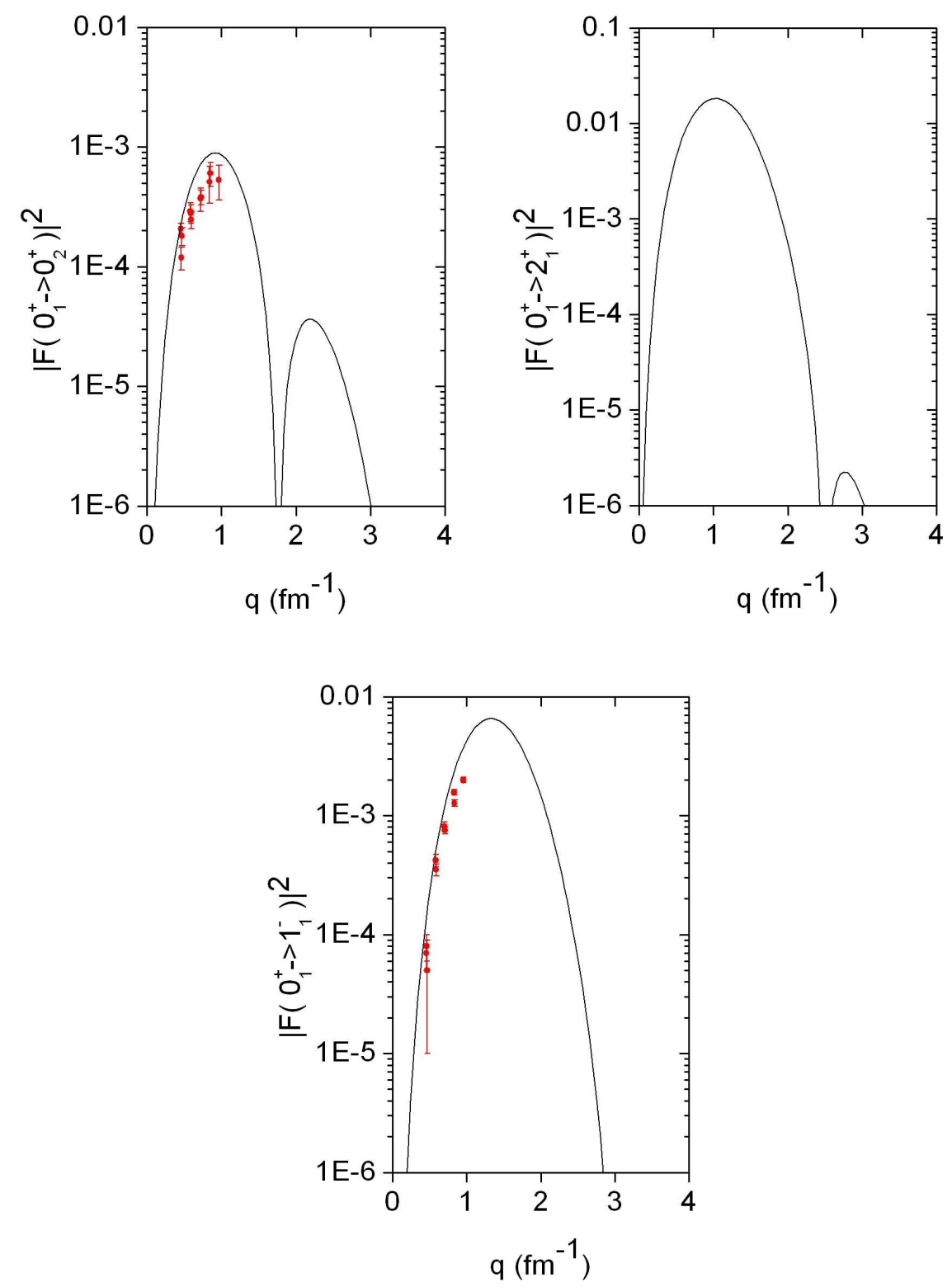

Figure 8: Comparison between the experimental form factors $\left|\mathcal{F}\left(0_{1}^{+} \rightarrow L_{i}^{P}\right)\right|^{2}$ of ${ }^{16} \mathrm{O}$ for the final states with $L_{i}^{P}=0_{2}^{+}, 2_{1}^{+}$and $1_{1}^{-}$and those obtained for the spherical top with $N=10$ and $R^{2}=1.0$. The experimental data are taken from Ref. [78]. 
Table 6: Comparison between experimental and theoretical values for transitions between the ground state and states in the vibrational bands. The experimental values are taken from [74].

\begin{tabular}{ccc}
\hline$B\left(E L ; L^{P} \rightarrow 0^{+}\right)$ & Th & $\operatorname{Exp}$ \\
\hline$M\left(0_{1}^{+} \rightarrow 0_{2}^{+}\right)$ & 0.54 & $3.55 \pm 0.21 \mathrm{fm}^{2}$ \\
$B\left(E 1 ; 1_{1}^{-} \rightarrow 0_{1}^{+}\right)$ & 0 & $(1.4 \pm 0.1) \times 10^{-4} \mathrm{e}^{2} \mathrm{fm}^{2}$ \\
$B\left(E 2 ; 2_{1}^{+} \rightarrow 0_{1}^{+}\right)$ & 26 & $7.4 \pm 0.2 \mathrm{e}^{2} \mathrm{fm}^{4}$ \\
\hline
\end{tabular}

Table 7: Comparison between experimental and theoretical $B(E 2)$ values in $\mathrm{e}^{2} \mathrm{fm}^{4}$. The experimental values are taken from [74].

\begin{tabular}{ccc}
\hline$B\left(E L ; L^{P} \rightarrow L^{\prime P^{\prime}}\right)$ & Th & Exp \\
\hline$B\left(E 2 ; 2_{1}^{+} \rightarrow 0_{2}^{+}\right)$ & 6 & $65 \pm 7$ \\
$B\left(E 2 ; 4_{1}^{+} \rightarrow 2_{1}^{+}\right)$ & 0 & $156 \pm 14$ \\
$B\left(E 2 ; 4_{2}^{+} \rightarrow 2_{1}^{+}\right)$ & 36 & $2.4 \pm 0.7$ \\
$B\left(E 2 ; 1_{1}^{-} \rightarrow 3_{1}^{-}\right)$ & 19 & $50.3 \pm 12.0$ \\
$B\left(E 2 ; 2_{1}^{-} \rightarrow 3_{1}^{-}\right)$ & 10 & $19.6 \pm 1.7$ \\
$B\left(E 2 ; 2_{1}^{-} \rightarrow 1_{1}^{-}\right)$ & 8 & $24.7 \pm 3.6$ \\
\hline
\end{tabular}

$I=0$. Since the transition operator $\hat{D}$ is a vector in index spin, the $B(E 2)$ value vanishes. The experimental value is large. On the other hand, the calculated $B\left(E 2 ; 4_{2}^{+} \rightarrow 2_{1}^{+}\right)$value is large. Therefore, either the assignment of $4_{1}^{+}$and $4_{2}^{+}$ should be interchanged, in which case an attempt should be made to measure the $B\left(E 4 ; 4_{2}^{+} \rightarrow 0_{1}^{+}\right)$, or the two states are strongly mixed due to their vicinity in energy as seen in Fig. 5.

The same situation occurs for the $2_{1}^{+}$and $2_{2}^{+}$states which we have assigned to (010) $E$ and $(001) F_{2}$. Therefore, while the assignments of the bandheads of the vibrational bands may be correct, it is not clear whether the states built on them are correctly assigned, a situation similar to that encountered in ${ }^{12} \mathrm{C}$ [31].

\section{Summary and conclusions}

In this paper, we have introduced an algebraic description of the four-body problem in terms of the spectrum generating algebra (SGA) of $U(10)$ based on the bosonic representation of the Jacobi vectors, $\vec{\rho}, \vec{\lambda}$ and $\vec{\eta}$. In particular, we have shown that the ACM for four-body clusters contains the spherical top with tetrahedral $\mathcal{T}_{d}$ symmetry as a special solution. The spherical top has been applied to the study of the spectrum, form factors and electromagnetic transition rates of the nuclear ${ }^{16} \mathrm{O}$, as composed of four $\alpha$-particles with tetrahedral symmetry. Evidence for this symmetry is particularly strong for the ground state band (000) $A_{1}$ with a rotational sequence $L^{P}=0^{+}, 3^{-}, 4^{+}, 6^{+}$, and weaker for the excited bands (100) $A_{1},(010) E$ and $(001) F_{2}$. 


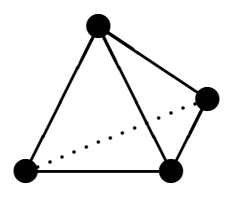

Tetrahedral: $\mathcal{T}_{d}$

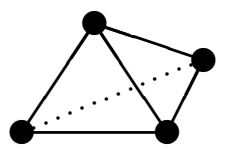

Pyramidal: $\mathcal{C}_{3 v}$

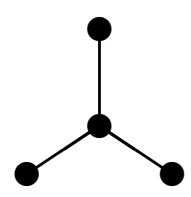

Planar: $\mathcal{D}_{3 h}$

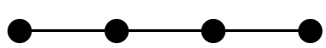

Linear: $\mathcal{C}_{\infty v}$

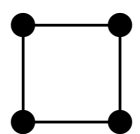

Square: $\mathcal{D}_{4 h}$

Figure 9: Four-body configurations: tetrahedral, pyramidal, planar, linear and square with their respective point-group symmetries.

The SGA of $U(10)$ can be used for any four-body problem whether the particles are identical or not, and for any discrete symmetry, be it $\mathcal{T}_{d}$ or other, and therefore it relevant for applications to other four-body problems in molecular, nuclear and hadronic physics, both for rigid and non-rigid configurations. Particularly simple are the descriptions of the rigid configurations of Fig. 9, tetrahedral, pyramidal and planar, and those of the harmonic oscillator $(U(9)$ limit) and the deformed oscillator $(S O(10)$ limit). Other configurations, such as the linear chain and the square are somewhat more involved, but they can be dealt with as well.

Finally, a variation of the algebraic method in which the Jacobi variables $\vec{\rho}$, $\vec{\lambda}$ and $\vec{\eta}$ are not fixed to their equilibrium values, as in the rigid configuration of Fig. 9, but are allowed to perform large amplitude motion can be used to describe quasi-molecular configurations. For $\alpha+{ }^{12} \mathrm{C}$, the variables $\vec{\rho}$ and $\vec{\lambda}$ of Fig. 1 need to be kept rigid as in our previous paper on ${ }^{12} \mathrm{C}$ [31], while the variable $\vec{\eta}$ may undergo large amplitude motion. A suitable algebraic description for these configurations is $U(7) \otimes U(4)$, where $U(7)$ describes the structure of ${ }^{12} \mathrm{C}$ and $U(4)$ the relative motion between $\alpha$ and ${ }^{12} \mathrm{C}[87]$.

\section{Acknowledgments}

This work was supported in part by research grant IN107314 from PAPIITDGAPA and in part by DOE Grant DE-FG02-91ER40608.

\section{References}

[1] J.A. Wheeler, Phys. Rev. 52 (1937), 1083. 
[2] L.R. Hafstad and E. Teller, Phys. Rev. 54 (1938), 681.

[3] D.M. Dennison, Phys. Rev. 96 (1954), 378.

[4] S.L. Kameny, Phys. Rev. 103 (1956), 358.

[5] D.M. Brink, Int. School of Physics "Enrico Fermi", Course XXXVI (1965), 247.

[6] D.M. Brink, H. Friedrich, A. Weiguny and C.W. Wong, Phys. Lett. B 33 (1970), 143.

[7] D. Robson, Nucl. Phys. A 308 (1978), 381.

[8] D. Robson, Prog. Part. Nucl. Phys. 8 (1982), 257.

[9] K. Wildermuth and Th. Kanellopoulos, Nucl. Phys. 7 (1958), 150.

[10] K. Ikeda, H. Horiuchi and S. Sato, Suppl. Progr. Theor. Phys. 68 (1980), 1.

[11] Y. Fujiwara, H. Horiuchi, K. Ikeda, M. Kamimura, K. Kato, Y. Suzuki and E. Uegaki, Suppl. Progr. Theor. Phys. 68 (1980), 29.

[12] H. Horiuchi, Prog. Theor. Phys. 51 (1974), 1266.

[13] H. Horiuchi, Prog. Theor. Phys. 53 (1975), 447.

[14] W. Von Oertzen, M. Freer and Y. Kanada-En'yo, Phys. Rep. 432 (2006), 43.

[15] M. Freer and H.O.U. Fynbo, Progr. Part. Nucl. Phys. 78 (2014), 1.

[16] M. Freer et al., Phys. Rev. C 76 (2007), 034320.

[17] O.S. Kirsebom et al., Phys. Rev. C 81 (2010) 064313.

[18] D.J. Marín-Lámbarri, R. Bijker, M. Freer, M. Gai, Tz. Kokalova, D.J. Parker and C. Wheldon, Phys. Rev. Lett. 113 (2014), 012502 [arXiv:1405.7445].

[19] M. Itoh et al., Phys. Rev. C 84 (2011), 054308.

[20] M. Freer et al., Phys. Rev. C 86 (2012), 034320.

[21] W.R. Zimmerman et al., Phys. Rev. Lett. 110 (2013), 152502.

[22] M. Freer et al., Phys. Rev. C 83 (2011), 034314.

[23] Y. Kanada-En'yo, Prog. Theor. Phys. 117 (2007), 655.

[24] M. Chernykh, H. Feldmeier, H. Neff, P. Von Neumann-Cosel and A. Richter, Phys. Rev. Lett. 98 (2007), 032501. 
[25] Y. Funaki, H. Horiuchi, W. Von Oertzen, G. Ropke, P. Schuck, A. Tohsaki and T. Yamada, Phys. Rev. C 80 (2009), 64326.

[26] R. Roth, J. Langhammer, A. Calci, S. Binder and P. Navrátil, Phys. Rev. Lett. 107 (2011), 072501.

[27] E. Epelbaum, H. Krebs, D. Lee and U.-G. Meissner, Phys. Rev. Lett. 106 (2011), 192501.

[28] E. Epelbaum, H. Krebs, T.A. Lähde, D. Lee and U.-G. Meissner, Phys. Rev. Lett. 109 (2012), 252501.

[29] A.C. Dreyfuss, K.D. Launey, T. Dytrych, J.P. Draayer and C. Bahri, Phys. Lett. B 727 (2013), 511.

[30] R. Bijker and F. Iachello, Phys. Rev. C 61 (2000), 067305.

[31] R. Bijker and F. Iachello, Ann. Phys. (N.Y.) 298 (2002), 334.

[32] R. Bijker and F. Iachello, Phys. Rev. Lett. 112 (2014), 152501.

[33] F. Iachello and A. Arima, The Interacting Boson Model (Cambridge U. Press, Cambridge, 1987).

[34] A. Arima and F. Iachello, Ann. Phys. (N.Y.) 99, 253 (1976).

[35] A. Arima and F. Iachello, Ann. Phys. (N.Y.) 111, 201 (1978).

[36] A. Arima and F. Iachello, Ann. Phys. (N.Y.) 123, 468 (1979).

[37] F. Iachello and R.D. Levine, Algebraic Theory of Molecules (Oxford U. Press, Oxford, 1995).

[38] F. Iachello, Chem. Phys. Lett. 78, 581 (1981).

[39] F. Iachello, in Lie Algebras, Cohomologies and New Applications of Quantum Mechanics, (N, Kamran and P. Olver, Eds.), Contemporary Mathematics, Vol. 160, p. 151, Amer. Math. Soc., Providence, RI, 1994.

[40] F. Iachello and A.D. Jackson, Phys. Lett. B 108 (1982), 151.

[41] F. Iachello, Nucl. Phys. A 396 (1983), 233c.

[42] H.J. Daley and F. Iachello, Phys. Lett. B 131 (1983), 281.

[43] F. Iachello, N.C. Mukhopadhyay and L. Zhang, Phys. Rev. D 44 (1991), 898.

[44] F. Iachello and D. Kusnezov, Phys. Rev. D 45 (1992), 4156.

[45] R. Bijker, F. Iachello and A. Leviatan, Ann. Phys. (N.Y.) 236 (1994), 69.

[46] R. Bijker, F. Iachello and A. Leviatan, Ann. Phys. (N.Y.) 284 (2000), 89. 
[47] R. Bijker, A.E.L. Dieperink and A. Leviatan, Phys. Rev. A 52 (1995), 2786.

[48] R. Bijker and A. Leviatan, Few-Body Systems 25 (1998), 89.

[49] R. Bijker, AIP Conf. Proc. 1323 (2010), 28.

[50] R. Bijker, J. Phys.: Conf. Ser. 380 (2012), 012003.

[51] R. Bijker, Phys. Scr. 91, 073005 (2016), 074006 [arXiv:1606.01306].

[52] P. Kramer and M. Moshinsky, Nucl. Phys. 82 (1966), 241.

[53] I. Talmi, Helv. Phys. Acta 25 (1952), 185.

[54] M. Moshinksy, Nucl. Phys. 13 (1959), 104.

[55] J. Dobeš, J. Phys. A: Math. Gen. 10 (1977), 2053.

[56] O.S. van Roosmalen and A.E.L. Dieperink, Ann. Phys. (N.Y.) 139 (1982), 198.

[57] S. Levit and U. Smilansky, Nucl. Phys. A 389 (1982), 56.

[58] G. Herzberg, Molecular Spectra and Molecular Structure. II. Infrared and Raman Spectra of Polyatomic Molecules (Krieger, Malabar Florida, 1991).

[59] T. Oka, J. Mol. Spectrosc. 48 (1973), 503.

[60] R. Bijker, computer program FFACM, unpublished.

[61] R. Bijker and J.N. Ginocchio, Phys. Rev. C 45 (1992), 3030.

[62] R. Bijker, Phys. Scr. 90 (2015), 074006 [arXiv:1412.5552].

[63] R. Bijker, R.D. Amado and D.A. Sparrow, Phys. Rev. A 33 (1986), 871.

[64] A.P. Zuker, B. Buck and J.B. McGrory, Phys. Rev. Lett. 21 (1968), 39.

[65] G.E. Brown and A.M. Green, Nucl. Phys. 75 (1966), 401.

[66] H. Feshbach and F. Iachello, Phys. Lett. B 45 (1973), 7.

[67] D. Robson, Phys. Rev. Lett. 42 (1979), 876.

[68] D. Robson, Phys. Rev. C 25 (1982), 1108.

[69] Y. Suzuki, Prog. Theor. Phys. 55 (1976), 1751.

[70] P. Navrátil, Proc. Int. School of Physics "Enrico Fermi", Course CLXIX [arXiv:0711.2702], and references therein.

[71] E. Epelbaum, H. Krebs, T.A. Lähde, D. Lee, U.-G. Meissner and G. Rupak, Phys. Rev. Lett. 112 (2014), 102501. 
[72] Y. Funaki, T. Yamada, H. Horiuchi, G. Röpke, P. Schuck and A. Tohsaki, Phys. Rev. Lett. 101 (2008), 082502.

[73] Y. Funaki, T. Yamada, A. Tohsaki, H. Horiuchi, G. Röpke and P. Schuck, Phys. Rev. C 82 (2010), 024312.

[74] D.R. Tilley, H.R. Weller and C.M. Cheves, Nucl. Phys. A 564 (1993), 1.

[75] D.M. Dennison, Rev. Mod. Phys. 12 (1940), 175.

[76] I. Sick and J.S. McCarthy, Nucl. Phys. A 150 (1970), 631.

[77] H. Crannell, Phys. Rev. 148 (1966), 1107.

[78] J.C. Bergstrom, W. Bertozzi, S. Kowalski, X.K. Maruyama, J.W. Lightbody Jr., S.P. Fivozinsky and S. Penner, Phys. Rev. Lett. 24 (1970), 152.

[79] J.C. Bergstrom, I.P. Auer, F.J. Kline and H.S. Caplan, Nucl. Phys. A 213 (1973), 609.

[80] M. Stroetzel, Z. Phys. 214 (1968), 357.

[81] G.R. Bishop, C. Betourne and D.B. Isabelle, Nucl. Phys. 53 (1964), 366.

[82] R.F. Frosch, J.S. McCarthy, R.E. Rand and M.R. Yearian, Phys. Rev. 160 (1967), 874 .

[83] I. Sick, J.S. McCarthy and R.R. Whitney, Phys. Lett. B 64 (1976), 33.

[84] J.S. McCarthy, I. Sick and R.R. Whitney, Phys. Rev. C 15 (1977), 1396.

[85] C.R. Ottermann, G. Köbschall, K. Maurer, K. Röhrich, Ch. Schmitt and V.H. Walther, Nucl. Phys. A 436 (1985), 688.

[86] R. Bijker, work in progress.

[87] F. Iachello, Phys. Rev. C 23 (1981), 2778. 